
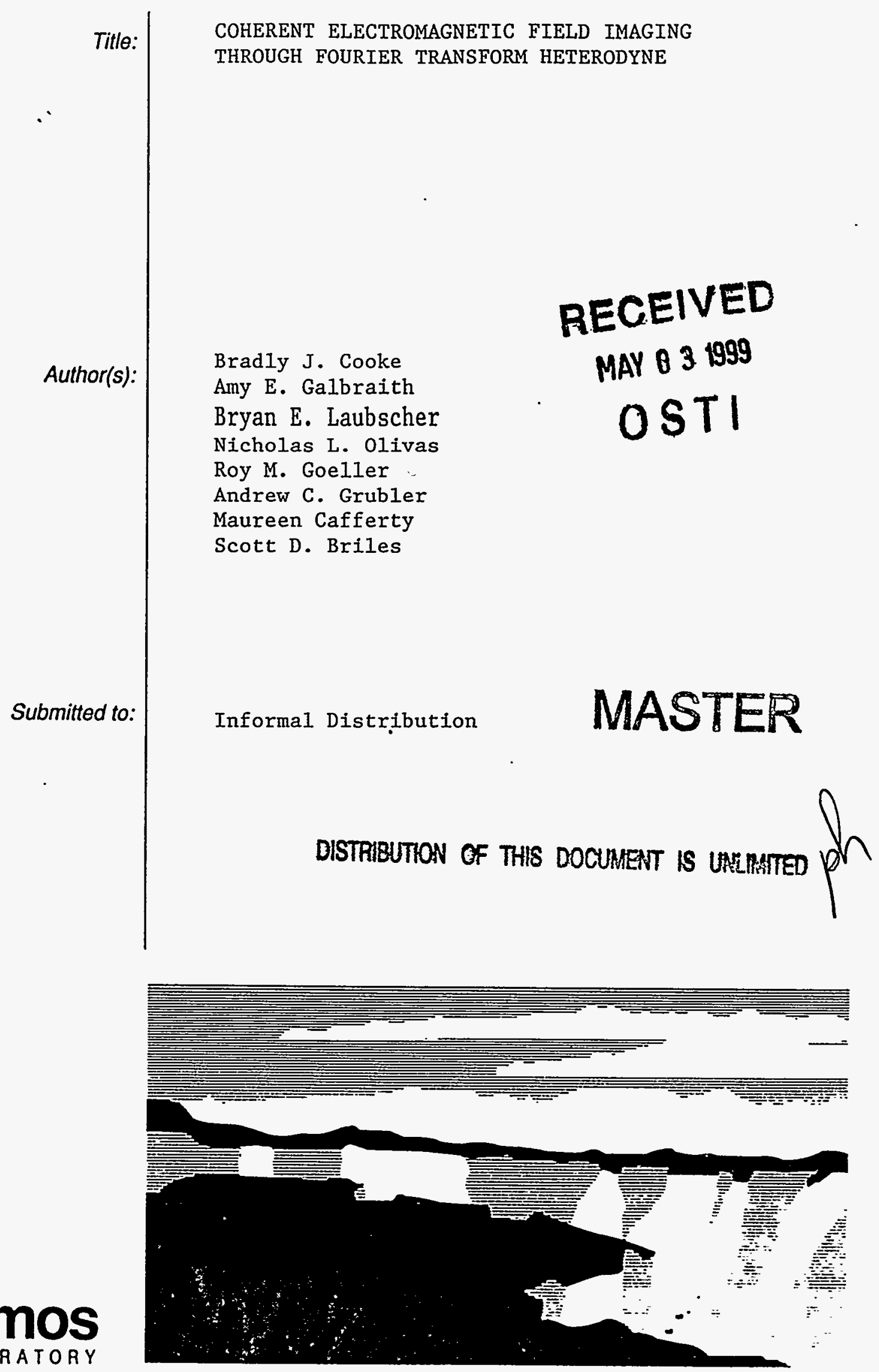

Los Alamos National Laboratory, an aftiemative actionequal opportunity empidyer. is operated by the University of Californla tor the U.S. Department of Enetoy undor coniract W-7405.ENG-36. By acceptanco of this articlo, the publishor recognizes that the U S. Government retains a nonexclusive. royalty-lroe license to publish or roproduce tho publishod torm of this contribution. or to allow othors to do so. for U.S. Government purposes. The Los Alamos National Laboratory requests that the pubtisher vontily this anticle as work performed under the auspices of tho U S. Dopartment of Enorgy 


\section{DISCLAIMER}

This report was prepared as an account of work sponsored by an agency of the United States Government Neither the United States Government nor any agency thereof, nor any of their employes, makes any warranty, express or implied. or assumes any legal liability or responsibility for the aceuracy, compieteness, or use. fulness of any information, apparatus, product, or process disclosed or represents that its use would not infringe privately owned rights. Reference berein to any specific commercial product, process, or service by trade name, tradernark, inanufacturer, or otherwise does aot neesessarily constitute or imply its endorsement. recommendation, or favoring by the United States Government or any agency thereof. The views and opinions of authors expressed herein do not necessarily state or reflect those of the United States Goverameat or any agency thereof. 


\section{DISCLAIMER}

Portions of this document may be illegible in electronic image products. Images are produced from the best available original document. 


\title{
COHERENT ELECTROMAGNETIC FIELD IMAGING THROUGH FOURIER TRANSFORM HETERODYNE
}

Bradly J. Cooke, Amy E. Galbraith ${ }^{\dagger}$, Bryan E. Laubscher, Nicholas L. Olivas, Roy M. Goeller, Andrew C. Grubler ${ }^{\ddagger}$, Maureen Cafferty, and Scott D. Briles

\author{
Los Alamos National Laboratory \\ P.O. Box 1663, MS-D448 \\ Los Alamos, NM 87545 \\ $505-667-8512$
}

\begin{abstract}
We present a detection process capable of directly imaging the transverse amplitude, phase, and if desired, Doppler shift of coherent electromagnetic fields. Based on coherent detection principles governing conventional heterodyned RADAR/LIDAR systems, Fourier Transform Heterodyne (FTH) incorporates transverse spatial encoding of the local oscillator for image capture. Appropriate selection of spatial encoding functions, or basis set, allows image retrieval by way of classic Fourier manipulations. Of practical interest:

(i) imaging is accomplished on a single element detector requiring no additional scanning or moving components, and (ii) a wide variety of appropriate spatial encoding functions exist that may be adaptively configured in real-time for applications requiring optimal detection. In this paper, we introduce the underlying principles governing FTH imaging, followed by demonstration of concept via a simple experimental setup based on a HeNe laser and a 69 element spatial phase modulator.
\end{abstract}

Key words: coherent electromagnetic field imaging, imaging RADARLIDAR, adaptive imaging.

\section{Introduction}

The ability to image both amplitude and phase of mm-RF through optical coherent electromagnetic fields enables interesting adaptations of current RADAR/LIDAR and communications technology $\&$ applications. Field imaging permits the correction of phase-front distortion imposed by target, atmosphere, and receiver optics. A coherent receiver capable of adaptively tracking and correcting atmospheric induced phase-front error allows the realization of large aperture, long-range communication systems. ${ }^{1}$ Real-time adaptive processing algorithms, prompted by direct transverse field encoding, might be put to use in the realization of novel RADAR/LIDAR systems queuing on a specific target(s) shape, size, velocity and trajectory.

\footnotetext{
'Electrical and Computer Enginecring Department, University of Arizona
}

' United States Naval Academy, P.O. Box 12939, Annapolis, MD 21412 


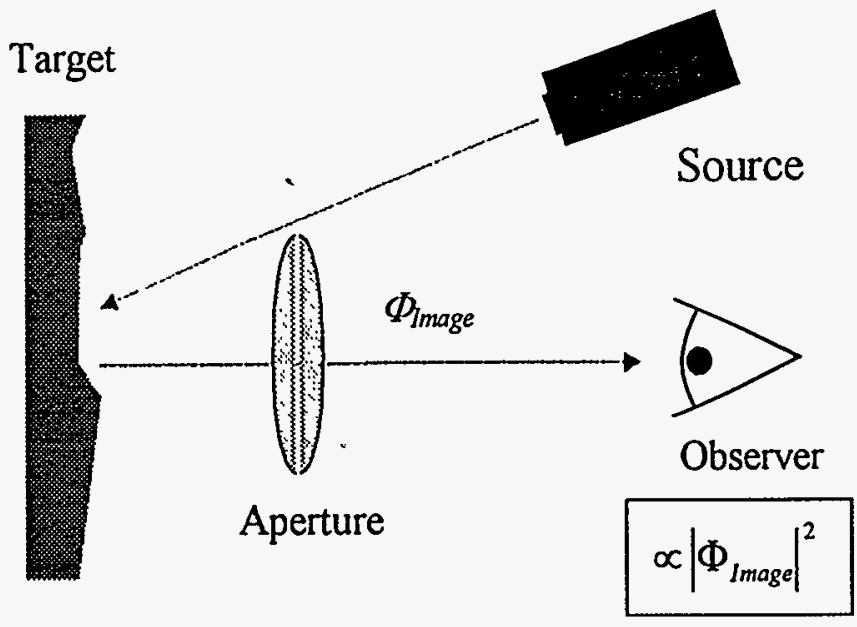

Figure 1. Basic square-law detection. A square-law detection process will result in the observer imaging the intensity of the field, with all phase information lost.
Electromagnetic field imaging requires a detection process in which both spatial amplitude and phase information is preserved. Eyes, film, CCD cameras, and other photosensitive elements image light in a process referred to as squared modulus or square-law detection. ${ }^{2}$ Referring to Figure 1, a coherent source such as a laser or RF transmitter illuminates a target to be imaged. The scattered image field, $\Phi_{\text {Image }}$, is collected at the receiving aperture and relayed to the observer. A square-law detection process will result in the observer imaging the intensity ${ }^{3}$ of the field,

$$
\mathbf{I}_{i} \propto\left|\Phi_{\text {Image }}\right|^{2},
$$

with all phase information lost. Preservation of both spatial amplitude and phase suggests a detection process that sidesteps the limitations imposed by the square-law operator.

In this paper, we introduce a heterodyned imaging detection process that circumvents the square-law limitations, and hence; is capable of directly imaging the transverse amplitude, phase, and if needed, the Doppler shift of coherent electromagnetic fields: Section 2 introduces the underlying principles governing FTH imaging, while Section 3 demonstrates the concept via a simple experimental setup based on a HeNe laser and a 69 element spatial phase modulator. Finally, practical considerations are discussed in Section 4.

\section{Fourier Transform Heterodyne}

Fourier Transform Heterodyne, or FTH, expands on the coherent detection principles governing conventional heterodyned RADAR/LIDAR systems, and incorporates transverse spatial encoding of the local oscillator for image retrieval. What follows in this section is a summary of the principle concepts underlying FTH. A more formal derivation is provided in Appendix A.

Referring to Figure 2, the observer depicted in Figure 1 has been replaced by the FTH system of interest. The basic steps involved in the capture of the image field, $\Phi_{\text {image, }}$, with the FTH detection process follow. 


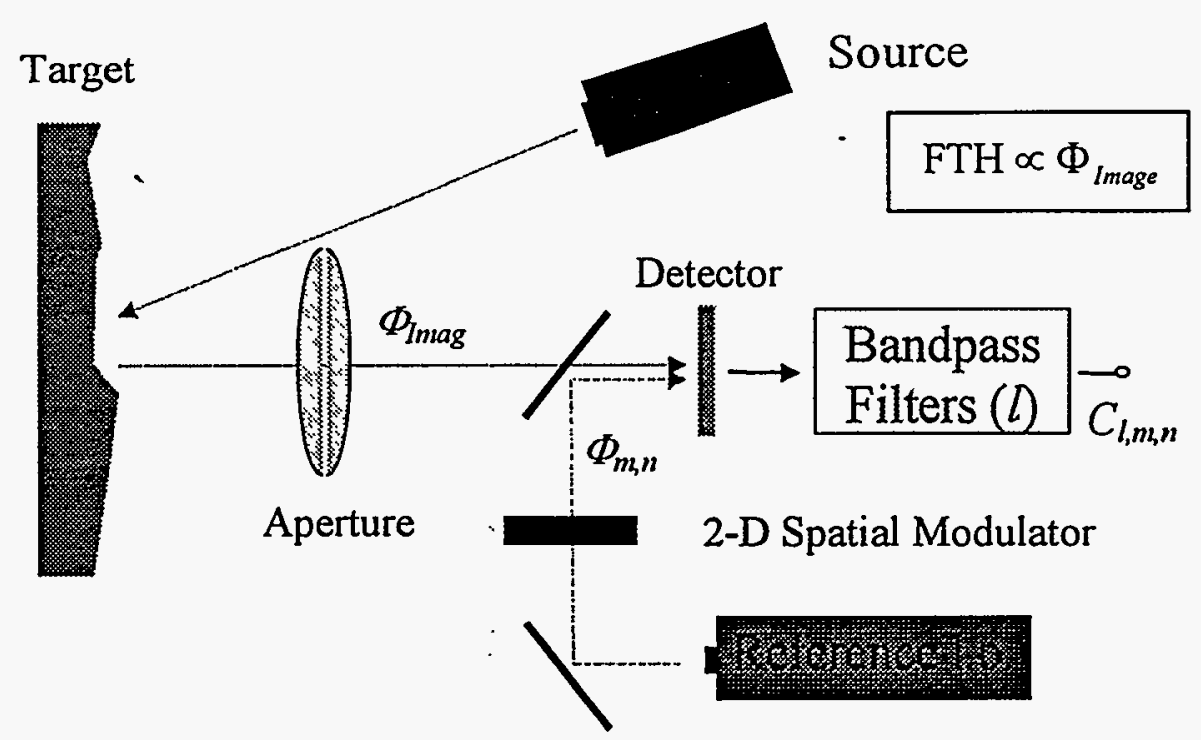

Figure 2. Fourier transform heterodyne (FTH). Based on coherent detection principles governing conventional heterodyned systems, FTH applies Fourier projection techniques through transverse spatial encoding $\left(\Phi_{m, n}\right)$ of the local oscillator for electromagnetic field imaging.

\section{FTH Field Imaging:}

- Heterodyne the image field, $\Phi_{\text {image }}$, with a local oscillator (l.o.) whose transverse amplitude and phase is sequentially modulated with a known set of reference spatial phase functions, $\Phi_{\text {Ref }}=$ $\Phi_{m, n}$, that form a valid basis set.

- Measure the magnitude and phase of the intermediate frequency (I.F.) detector current and form a complex Fourier coefficient, $C_{l, m, n}$, for each spatial function, $\Phi_{m, n}$. As will be derived presently, the heterodyned square-law detection process can be perceived as a Fourier projection operator with the I.F. current conveying the magnitude and phase of the projection. The index $l$ represents the Doppler index of the center frequency $(\Delta \omega)$ of the $l^{t h}$ bandpass filter.

- Reconstruct the image field from the basis functions, $\Phi_{m, n}$, and the measured $C_{l, m, n}$ :

$$
\Phi_{l m a g e}\left(x, y, \Delta \omega_{l}\right)=\Phi_{\text {Image }}(x, y, l)=\sum_{m} \sum_{n} C_{l, m, n} \Phi_{m, n}(x, y)
$$

The Fourier projection properties inherent to heterodyned square-law detection processes are subsequently summarized. From Figure 2, the dependence of the induced detector current, $I$, given the detector's quantum efficiency, $\eta$, and the spatial distribution of the image and reference local-oscillator electromagnetic fields, $\Phi_{\text {Image }}$ and $\Phi_{\text {Ref }}$ respectively, over the detector surface, $s$, is evaluated through Poynting's relation ${ }^{4,5,6}$ 
[ZI]

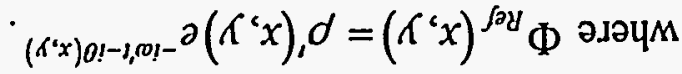

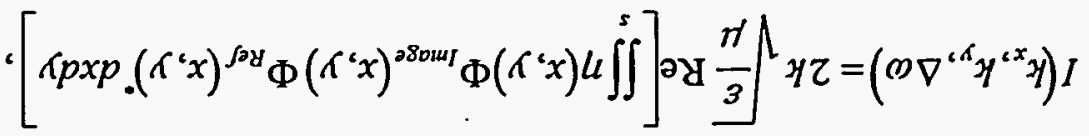

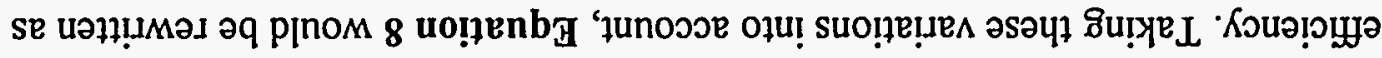

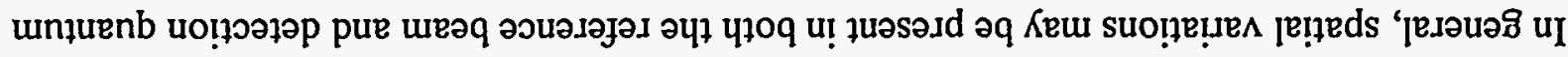

[oI]

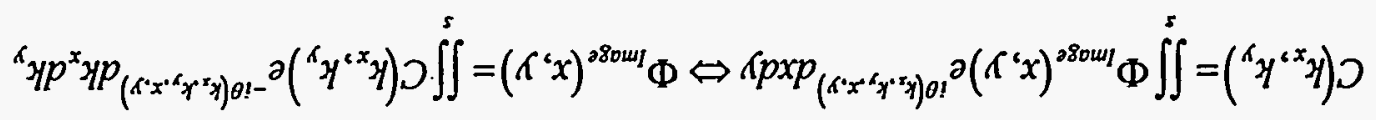

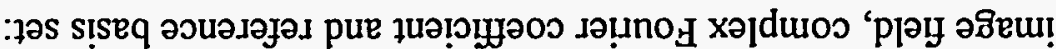

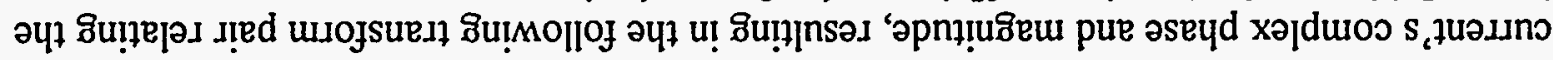

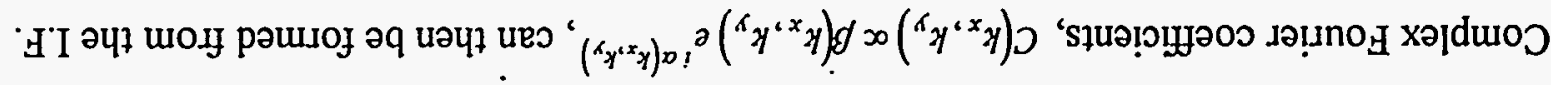

[6]

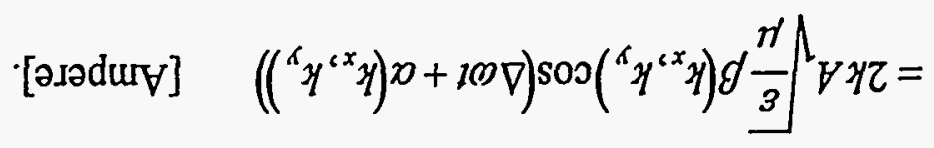

[8]

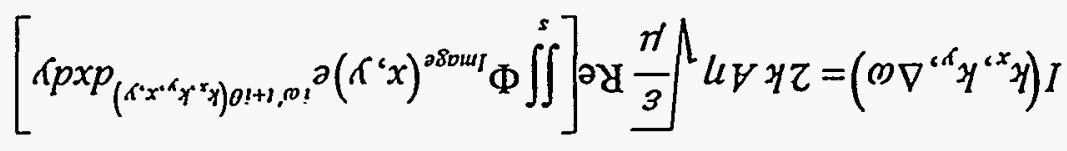

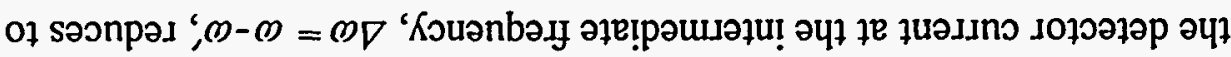

[L]

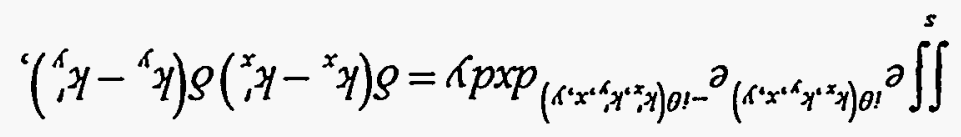

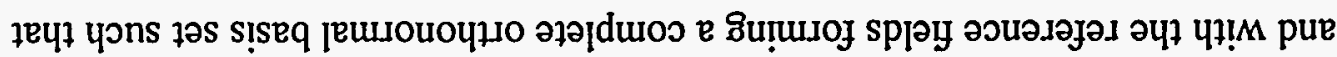

[9]

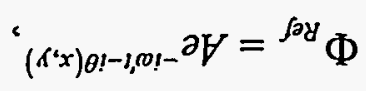

[s]

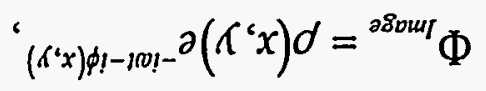

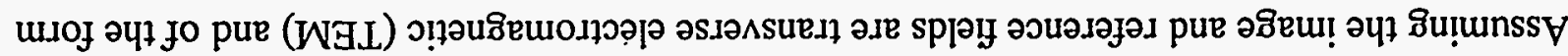
[b]

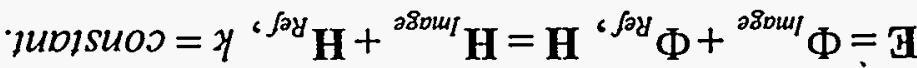

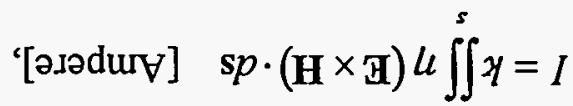


Finally, if the image field is heterodyned with a discrete set of reference fields $\left(\Phi_{R e f} \rightarrow \Phi_{m, n}\right)$, then Equation 11 reduces to the following Fourier expansion pair

$$
\begin{gathered}
\Phi_{\text {lmage }}\left(x, y, \Delta \omega_{l}\right)=\Phi_{\text {lmage }}(x, y, l)=\sum_{m} \sum_{n} C_{l, m, n} \Phi_{m, n}(x, y), \\
C_{l, m, n} \Rightarrow I_{l, m, n} \propto \iint_{s} \Phi_{l m a g e} \Phi_{m, n}^{*} d x d y,
\end{gathered}
$$

where the Doppler index $l$, representing the center frequency of the $l^{h}$ I.F. bandpass filter, has been included.

Note that the detecting surface, $s$, can be either a single element or partitioned into multiple elements and coherently summed,

$$
\iint_{s} \Phi_{I m a g e} \Phi_{m, n}^{*} d x d y \Rightarrow \sum_{s_{1}} \iint_{s_{i}} \Phi_{l m a g e} \Phi_{m, n}^{*} d x d y
$$

This property is of practical interest in the development of synthetic apertures and very-large-array (VLA) receivers.

\section{Experiment}

A simple FTH experiment designed to demonstrate the FTH concept has been constructed. Schematically illustrated in Figure 3 with photographed apparatus in Figure 4, the experiment implements a Zeeman split. HeNe laser $(\Delta f=250 \mathrm{kHz})$ for source and local-oscillator lines, and a 69 element spatial phase modulator to encode the 36 term basis used in the experiment.

A description of the FTH experimental procedure follows:

- The two orthogonally polarized laser lines $(\Delta f=250 \mathrm{kHz})$ at the output of the HeNe laser are split with the polarizing beam splitter into two separate beams paths.

- The first beam illuminates the target forming the image, $\Phi_{\text {Image, }}$, while the second beam passes through the 69-element spatial modulator generating the $n^{\text {th }}$ reference term, $\Phi_{n}, n=0,1, \ldots, 35$.

- The two beams are recombined and are heterodyned at the single-element silicon detector generating a $250 \mathrm{kHz}$ intermediate frequency current $I_{n}$.

- A digital oscilloscope measures the $250 \mathrm{kHz}$ I.F. current's phase and magnitude from which the Fourier coefficients, $C_{n}$, are formed (see Figure 5).

- Once all 36 basis coefficients, $C_{n}, n=0,1, \ldots, 35$, are sequentially measured, the resulting magnitude and phase of the detected image, $\Phi_{\text {Image }}$, are displayed through Equation 13 on the computer screen. 


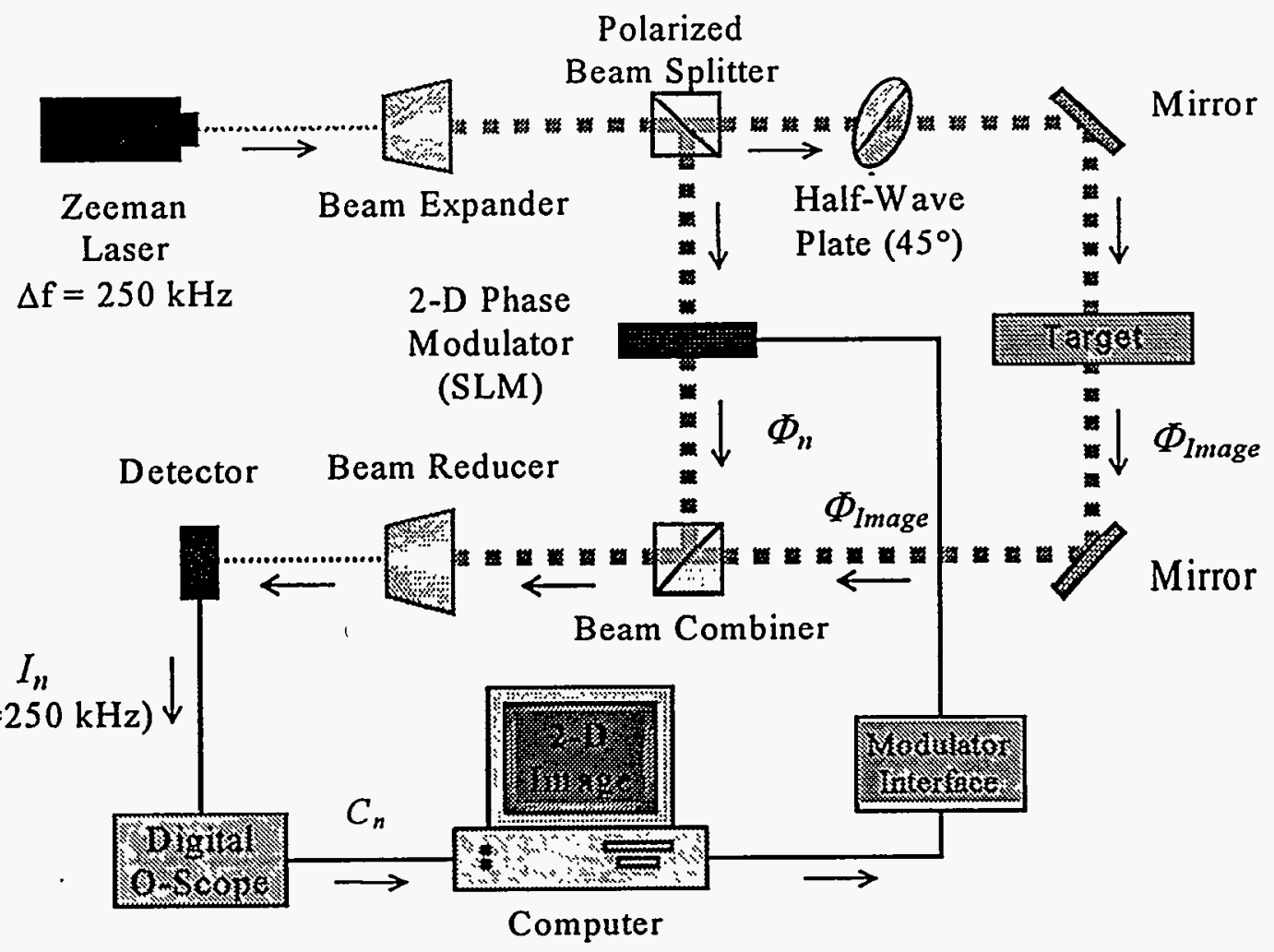

Figure 3. Schematic diagram of FTH experiment.

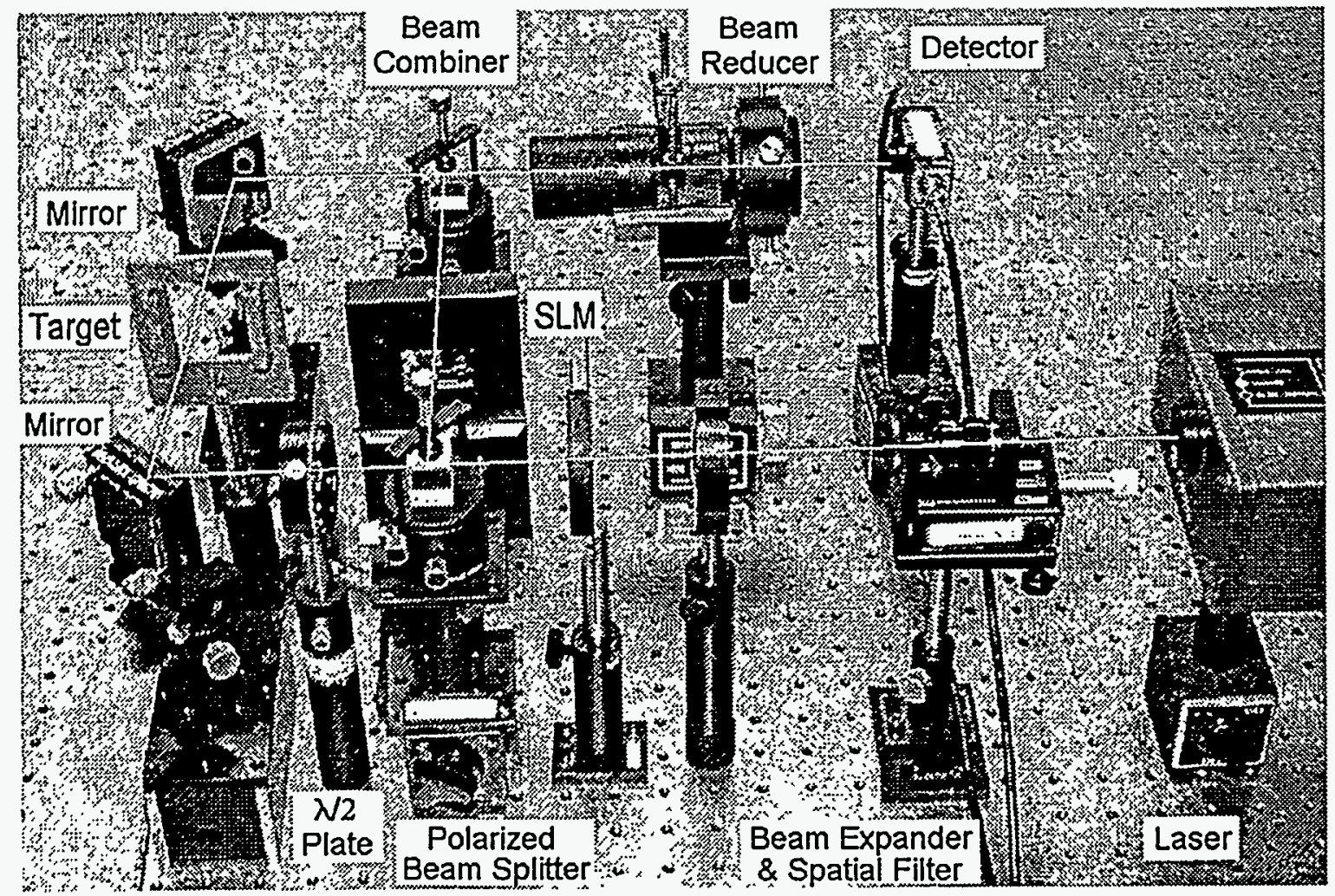

Figure 4. Experimental apparatus. 


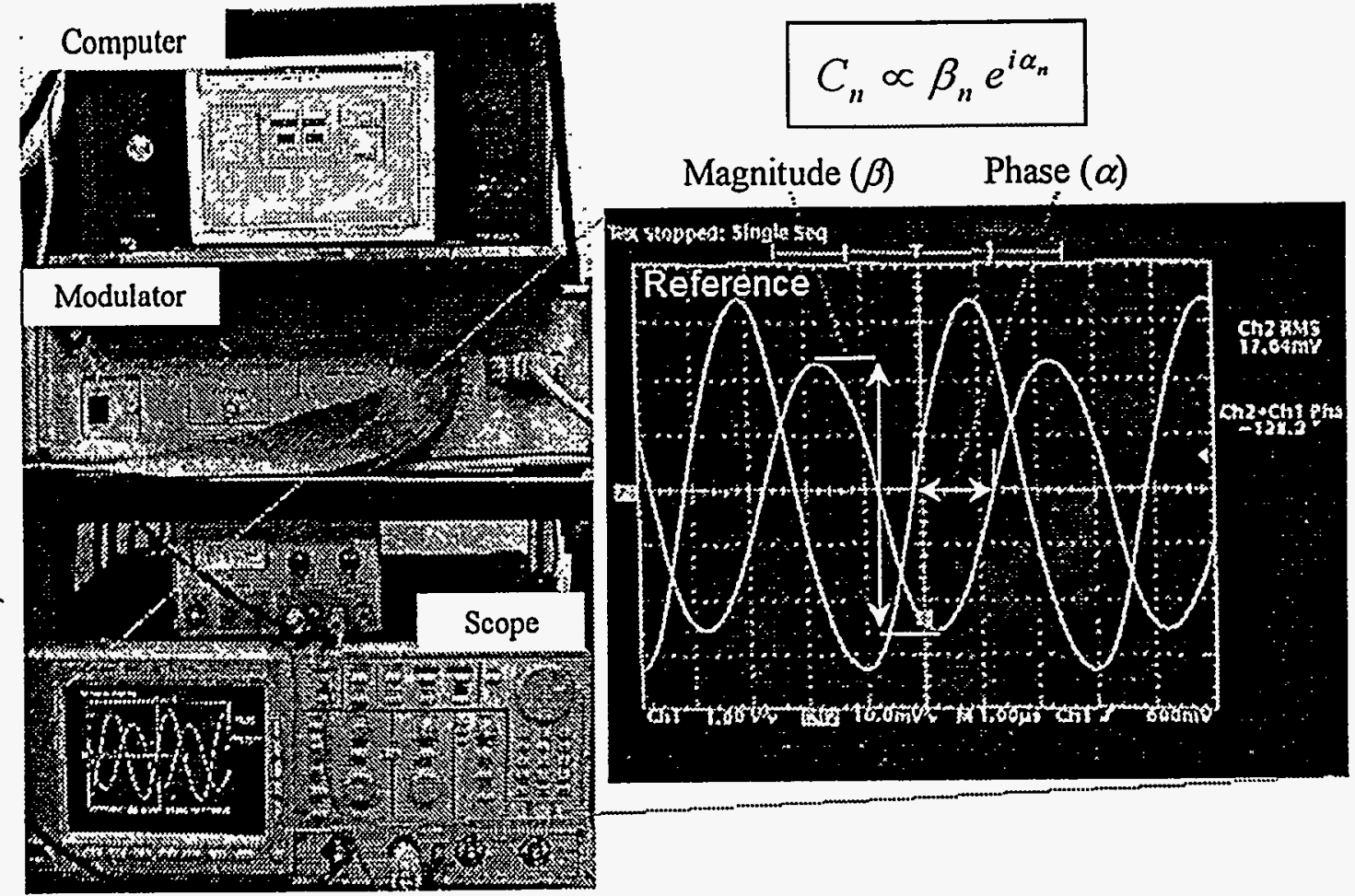

Figure 5. A digital oscilloscope measures the $250 \mathrm{kHz}$ I.F. current's phase and magnitude from which the Fourier coefficients, $C_{m}$, are formed.

The 2-D spatial phase modulator, implemented to encode the reference beam phase front, is the commercially produced Meadowlark Optics Hex69 spatial light modulator (SLM). The Meadowlark SLM is a 69 pixel, two-dimensional array of hexagon shaped, liquid-crystal variable retarders developed for real time programmable phase masking applications. As Meadowlark Optics conveniently supplies the software to generate the first 36 terms of the Zernike ${ }^{7}$ polynomial expansion set, the Zernike set was adopted for the experiment and Equation 12, in circular coordinates, takes on the form

$$
\Phi_{r e f} \rightarrow \Phi_{n}=\rho^{\prime}(r, \theta) e^{-i \omega^{\prime} t-i 2 \pi z_{n}(r, \theta)}, \quad n=0,1, \ldots, 35
$$

with the full $36 Z_{n}$ (Zernike polynomials) listed in Appendix B. Figure 6 catalogues the set of beam targets employed in the experiment. Note that commensurate with the rather limited 36 term expansion set, simple target geometry was observed during the experiment.
A)
B)
C)
D)
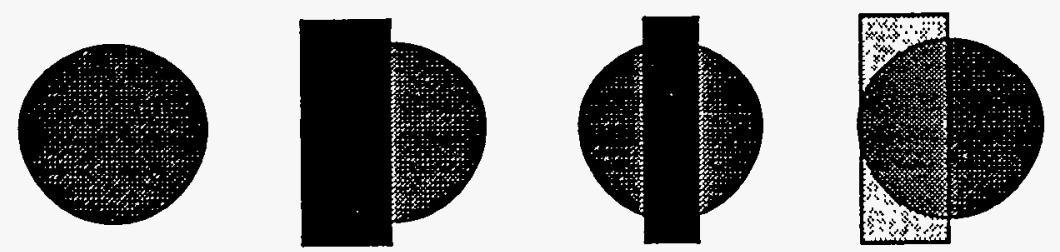

Figure 6. Beam targets: A) No target, B) $1 / 2$ Block, C) $1 / 3$ Center Block. D) $1 / 2$ Dielectric Block (glass microscope slide with transmission $T=90 \%$ and phase shift $\Delta 0=0.9 \pi$ ). 

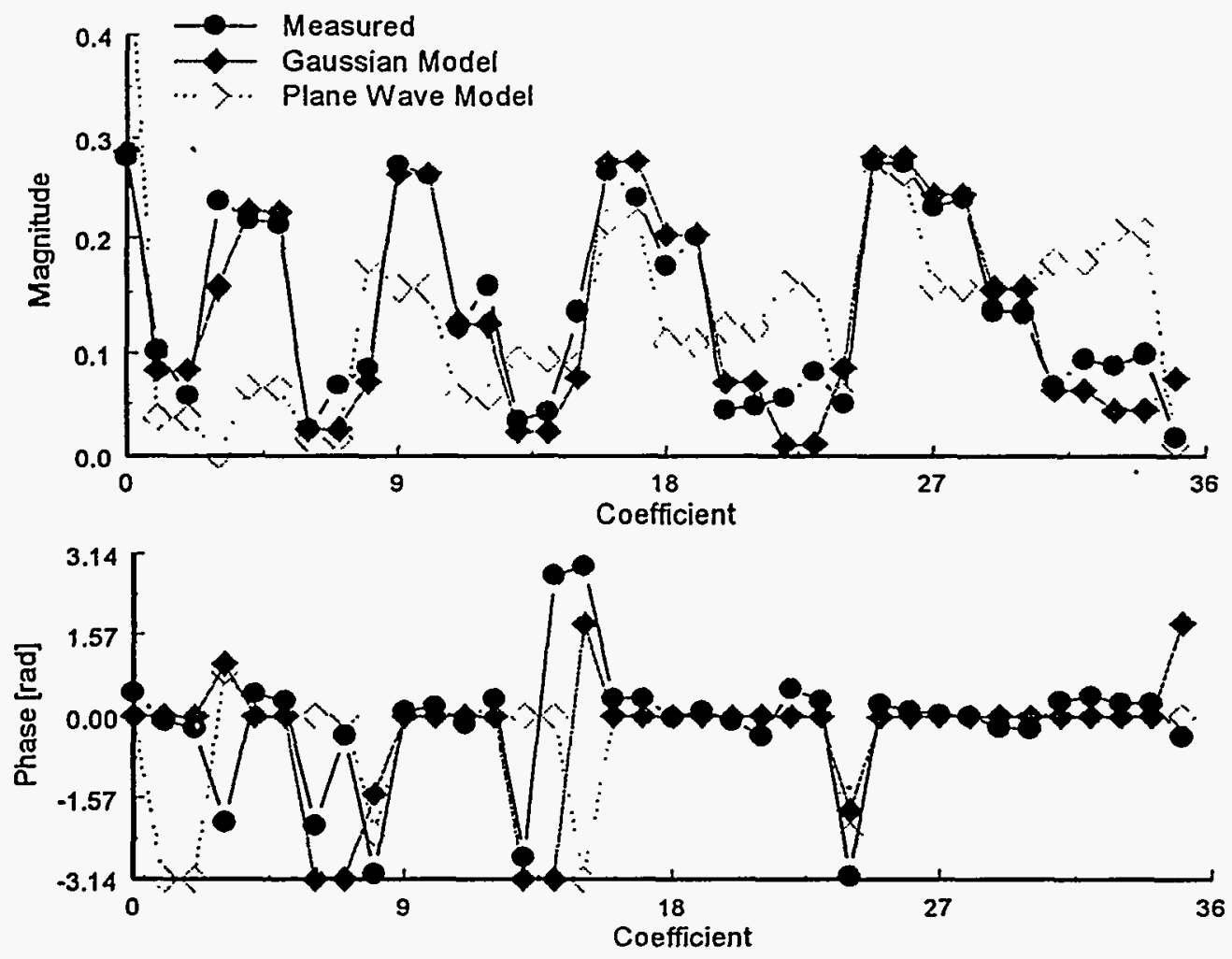

Figure 7. Measured spectral coefficients for no target. Included are plane-wave and Gaussian $(\omega=1 / 2)$ coefficients for baseline comparison (coefficients have been normalized) with the Gaussian model providing the best fit.

For the purpose of providing a comparative baseline between measurement and theory, the complex coefficients were directly evaluated from Equation 14, giving

$$
C_{n}=\int_{0}^{2 \pi} \int_{0}^{1} T(r, \theta) \rho(r, \theta) \rho^{\prime}(r, \theta) e^{2 \pi i Z_{n}(r .0)} r d r d \theta, \quad n=0,1, \ldots, 35,
$$

with:

$$
\begin{aligned}
& T(r, \theta) \equiv \text { beam targets of Figure } 6, \\
& \rho(r, \theta)=\rho^{\prime}(r, \theta)=1 \text { for a plane wave, and } \\
& \rho(r, \theta)=\rho^{\prime}(r, \theta)=e^{-\frac{r^{2}}{\omega^{2}}} \text { for a simple Gaussian beam }{ }^{8} .
\end{aligned}
$$

Plotted in Figure 7 are measured spectral coefficients (no target) along with the calculated spectral coefficients for the simple plane wave and Gaussian $(\omega=1 / 2)$ models. As it provided the best fit, the Gaussian model was evaluated for the remaining targets of Figure 6, generating baseline comparison with measurements. 
The theoretically predicted and experimentally measured amplitude, phase, and intensity (energy) for the beam targets of Figure 6 are plotted in Figures 8-11. Both calculated and measured coefficients were normalized and a constant phase offset was added to the measured coefficients to equalize pedestal/piston - no other data processing was introduced. From Equation 13, the field image was expanded with

$$
\Phi_{\text {lmage }}(r, \theta)=\sum_{n} C_{n} \Phi_{n}(r, \theta), \quad n=0,1, \ldots, 35,
$$

and the intensity

$$
\operatorname{Intensity}(r, \theta)=\left|\Phi_{\text {lmage }}(r, \theta)\right|^{2}
$$

It is important to remember that the primary purpose of the experiment is the demonstration of the underlying principles governing FTH imaging. Images shown in Figures 8-11 do indeed indicate that the basic mechanisms expected, such as Fourier projection, are present and valid. However, care should be taken when interpreting image content as the actual image essentially is filtered through the relatively low-resolution 36-term expansion window. Sharp amplitude and phase structure in the imaged field tends to excite energy into higher spatial modes that may fall outside of the 36-term window and result in image distortion (see Appendix C).

An urifortunate consequence of raising the Zernike polynomials to an exponent, as prescribed by Equation 12, is reflected in the fact that the 36-term basis set is no longer orthogonal. A nonorthogonal basis set will result in the off diagonal coupling of the expansion coefficients which, depending on coupling strength and image spectrum, can in turn degrade image fidelity. The magnitude of off diagonal coupling of Equation 16 is on the order of a rather high $5 \%-10 \%$. The coefficients can be decoupled through the following linear transform ${ }^{9}$

$$
\mathrm{C} \rightarrow \Lambda^{-1} \mathrm{C} \text {, }
$$

where:

$$
\begin{aligned}
& \mathbf{\Lambda}=\left[\begin{array}{ccc}
\left\langle Z_{0}, Z_{0}\right\rangle & \cdots & \left\langle Z_{0}, Z_{3 s}\right\rangle \\
\vdots & \ddots & \vdots \\
\left\langle Z_{3 s}, Z_{0}\right\rangle & \cdots & \left\langle Z_{3 s}, Z_{35}\right\rangle
\end{array}\right] \\
& \text { and }\left\langle Z_{p}, Z_{q}\right\rangle=\int_{0}^{2 \pi} \int_{0}^{1} e^{-2 \pi i Z_{p}(r, \theta)} e^{2 \pi i Z_{q}(r . \theta)} r d r d \theta .
\end{aligned}
$$

The transform is sensitive to systematic errors including source coherence, pixel distribution and geometry, and SLM phase drift that introduce deviations from the ideal basis set of Equation 16 (a strong endorsement for the use of orthogonal basis sets), and hence difficult to apply in practice. However, as shown in Figure 12, the transform was successful in bringing out the phase structure of Figure 11. Within the limitations imposed by the 36-term expansion, the formation of the phase shift discontinuity caused by the microscope slide edge is visible down the center of the phase image. 


\section{A) Gaussian Model}

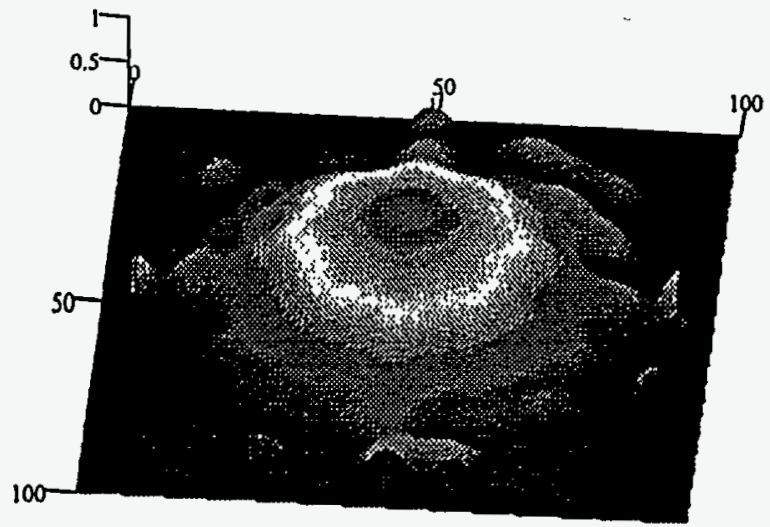

Amplitude

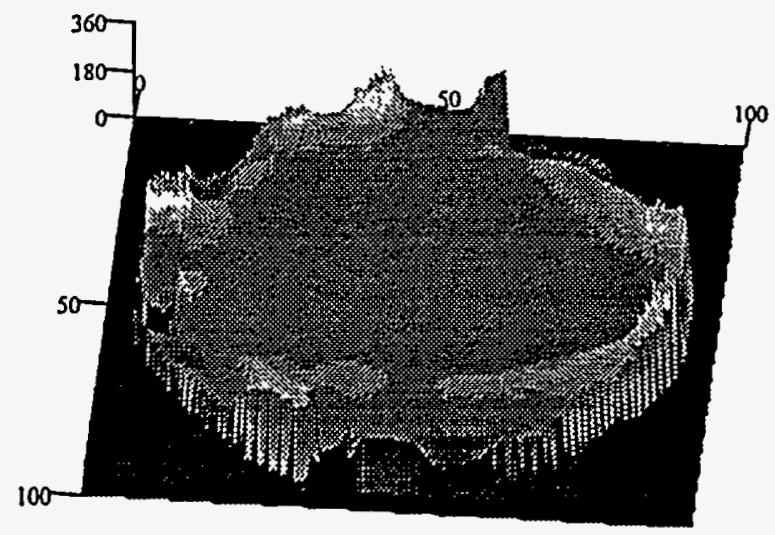

Phase

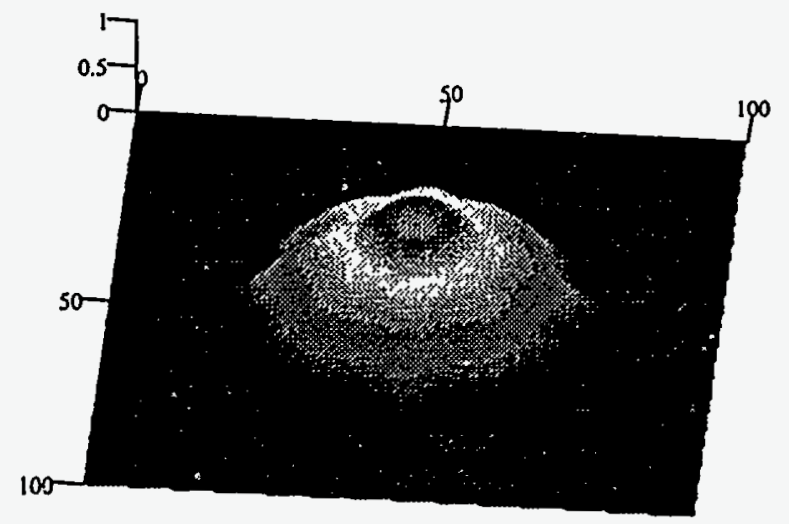

Intensity

\section{B) Measured}

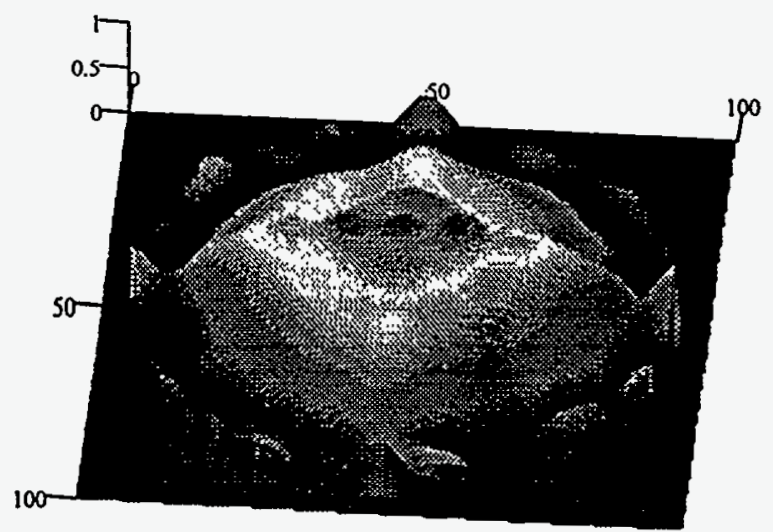

Amplitude

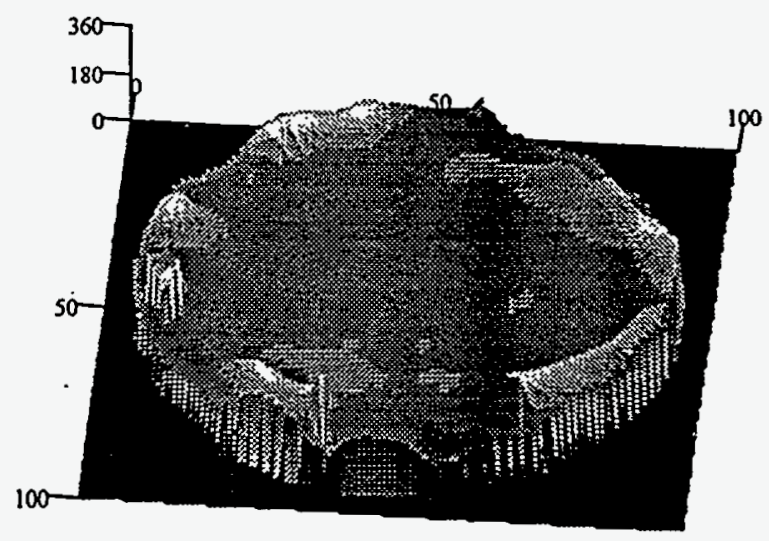

Phase

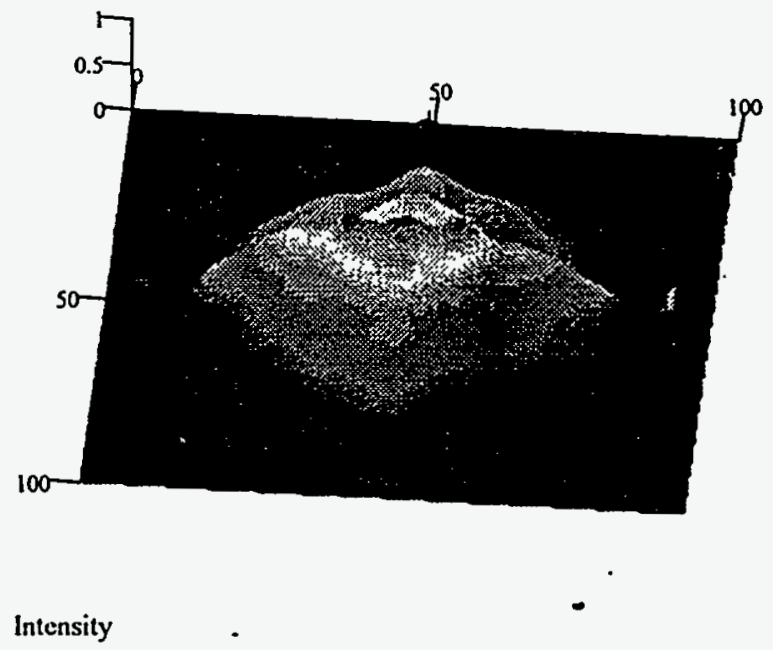

Figure 8. No target. A) Theoretically predicted and B) experimentally measured amplitude, phase, and intensity. 
A) Gaussian Model

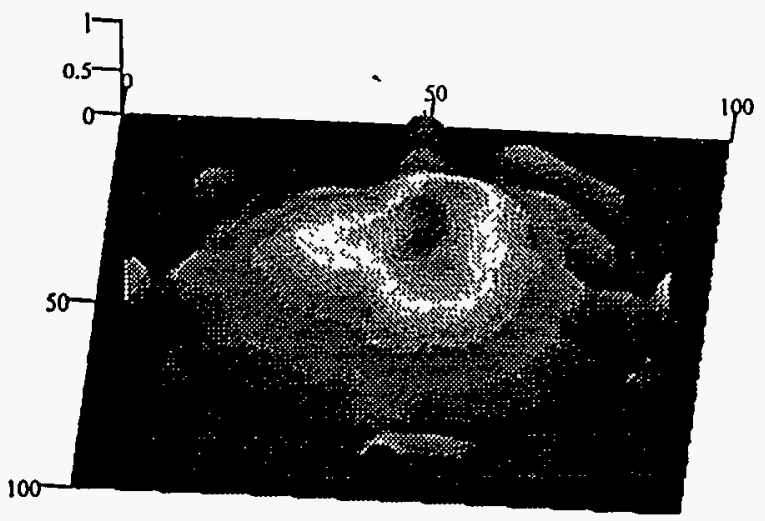

Amplitude

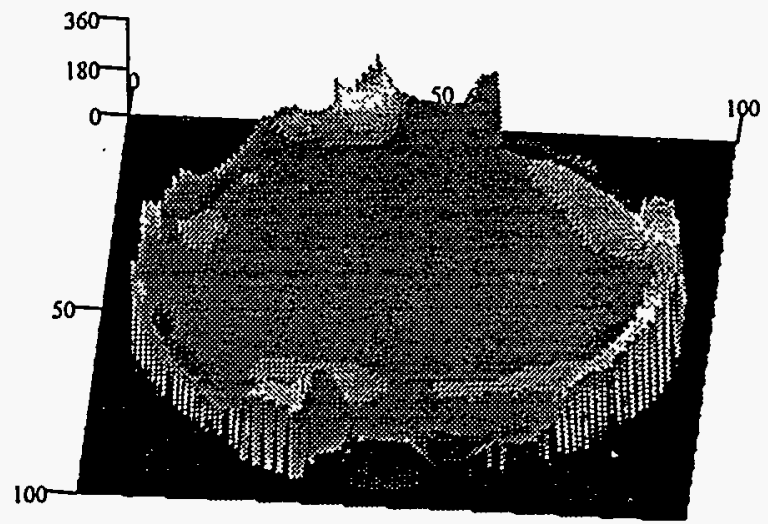

Phase

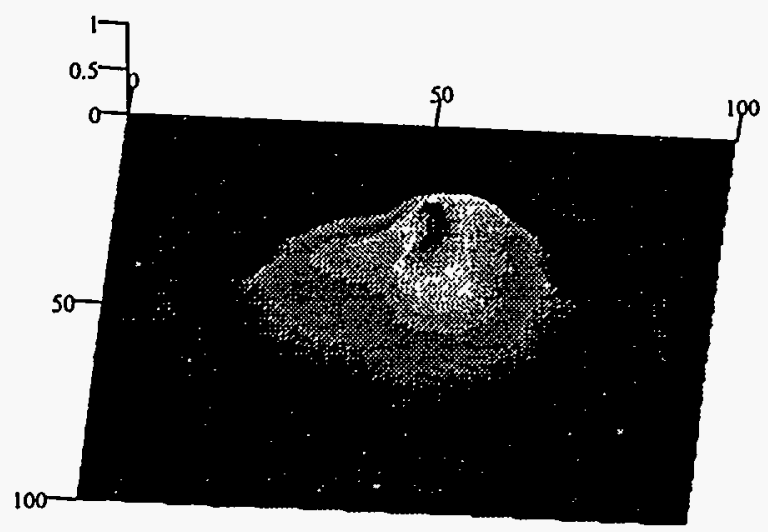

Intensity
B) Measured

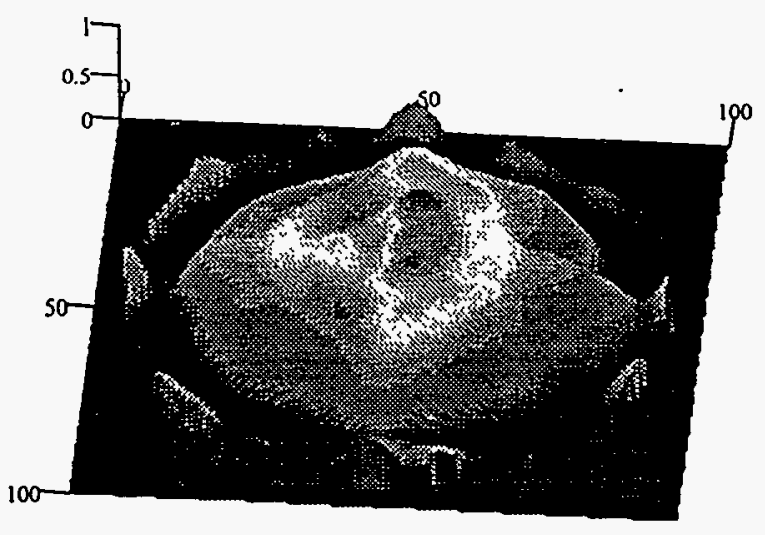

Amplitude

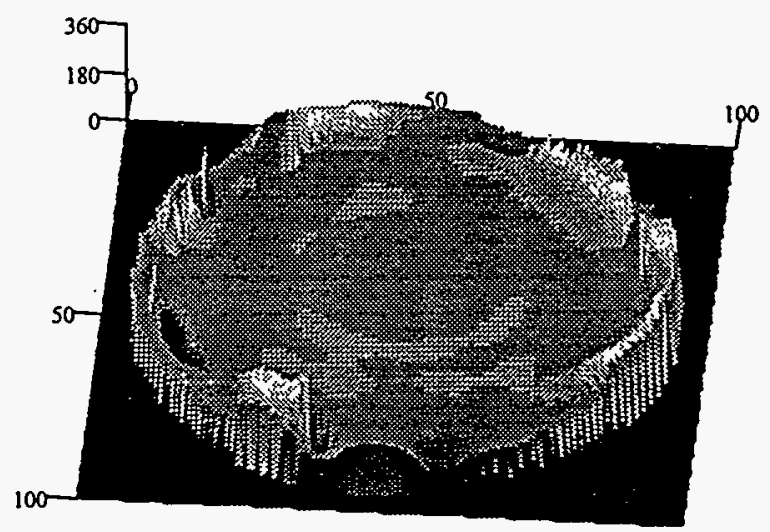

Phasc

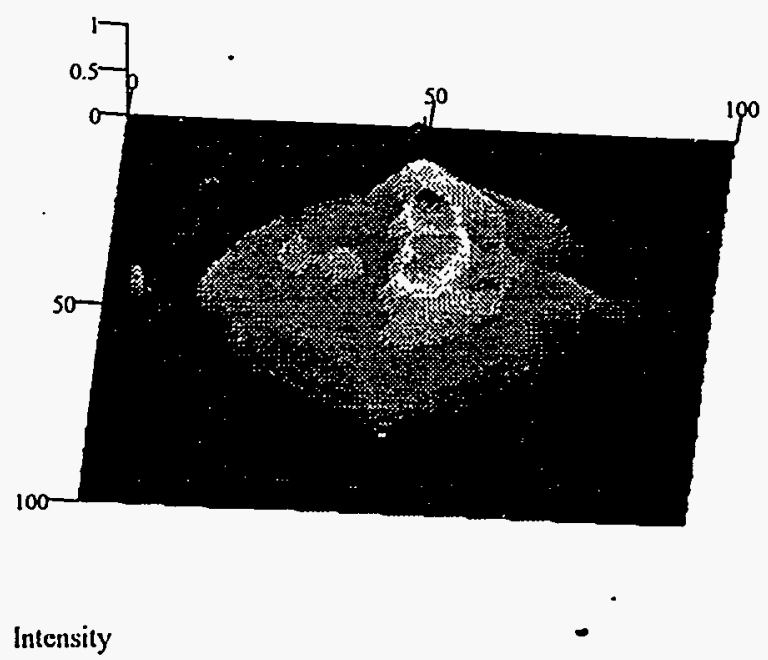

Figure 9. 1/2 Block. A) Theoretically predicted and B) experimentally measured amplitude, phase, and intensity. 


\section{A) Gaussian Model}

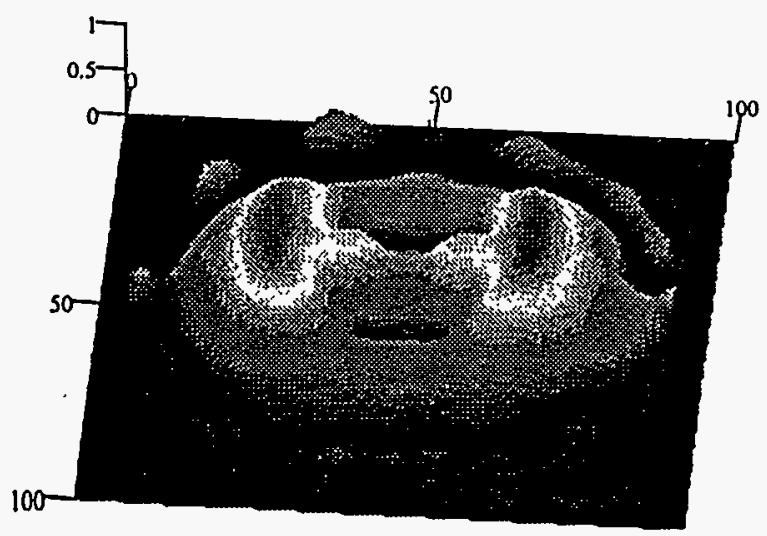

Amplitude

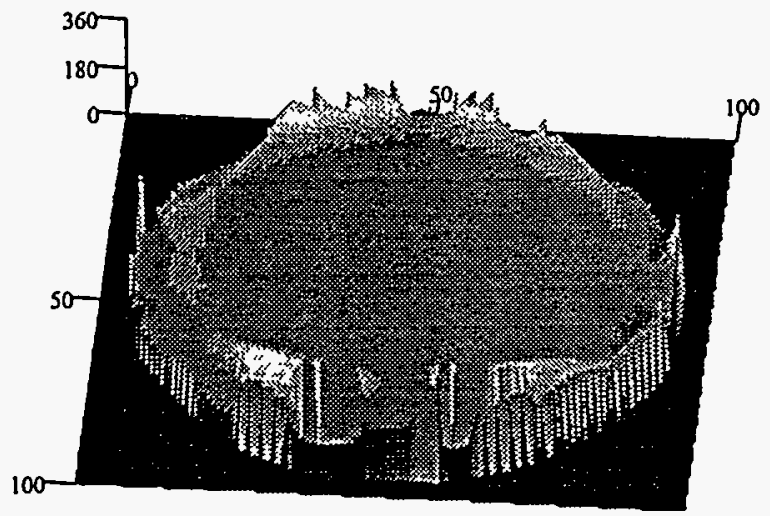

Phase

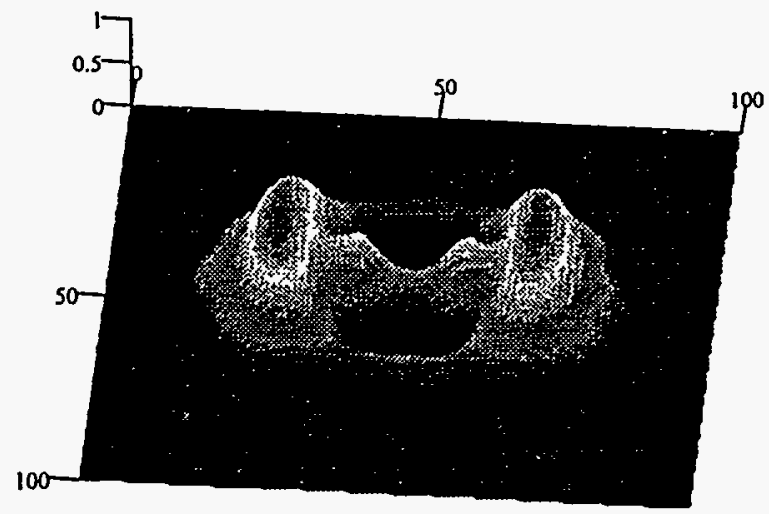

Intensity
B) Measured

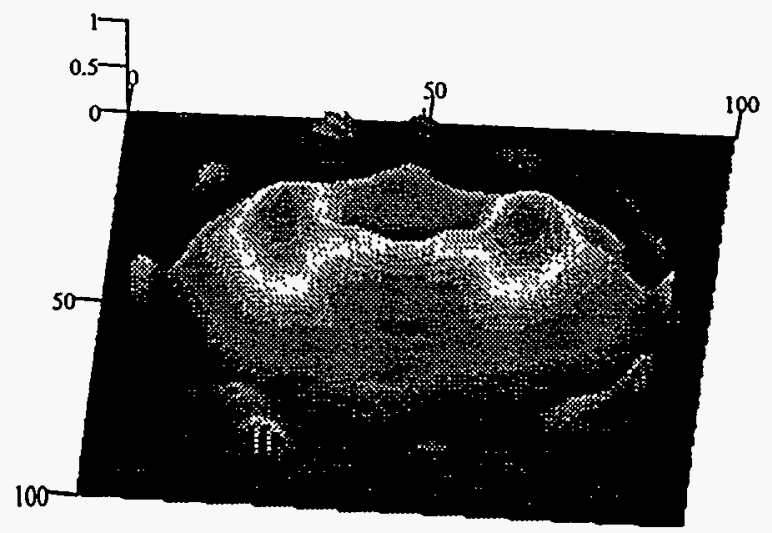

Amplitude

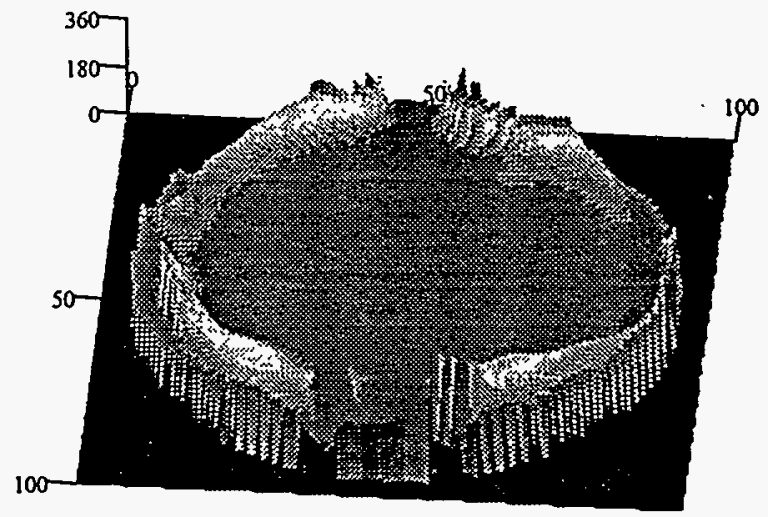

Phase

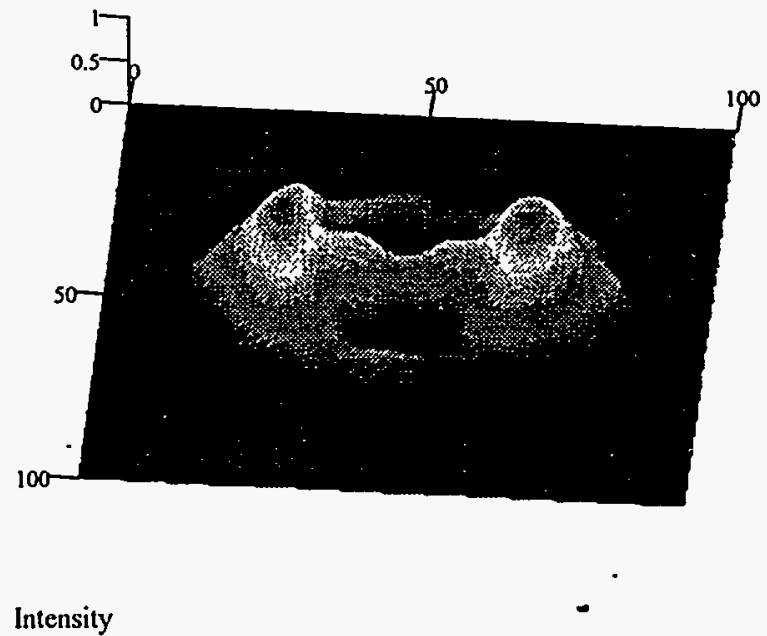

Figure 10. 1/3 Center Block. A) Theoretically predicted and B) experimentally measured amplitude, phase, and intensity. 
A) Gaussian Model

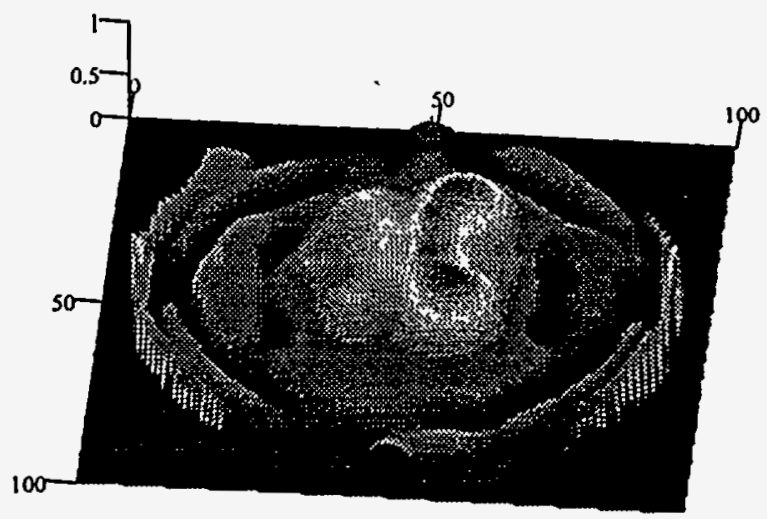

Amplitude

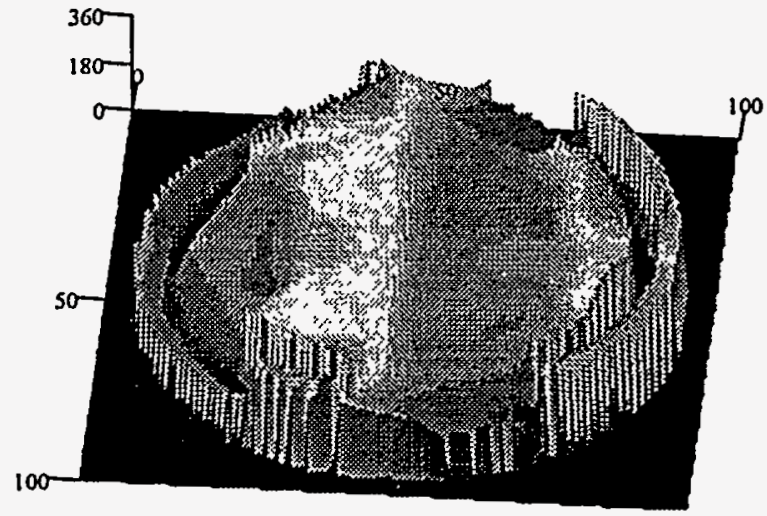

Phase

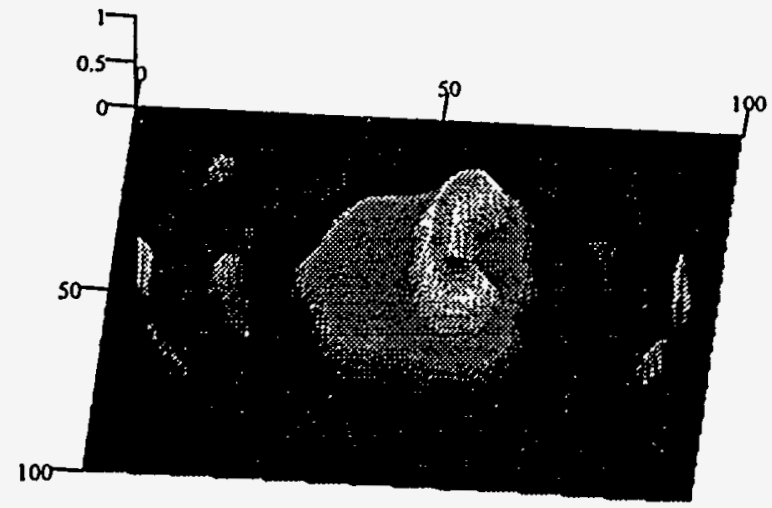

Intensity
B) Measured

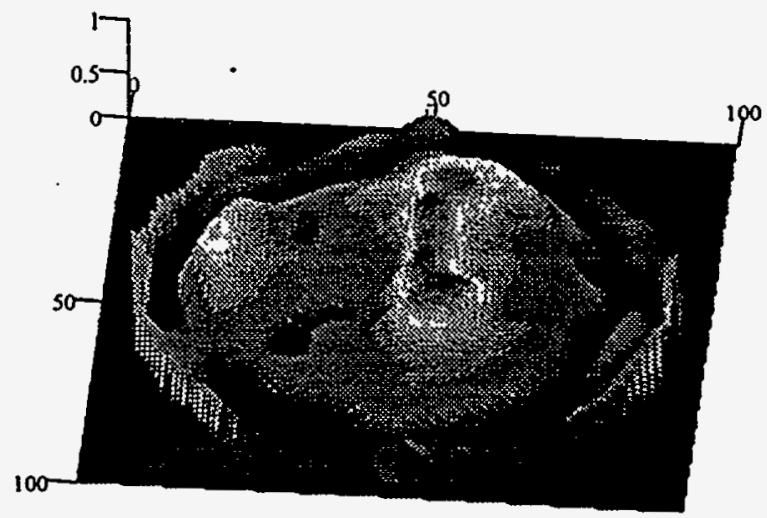

Amplitude

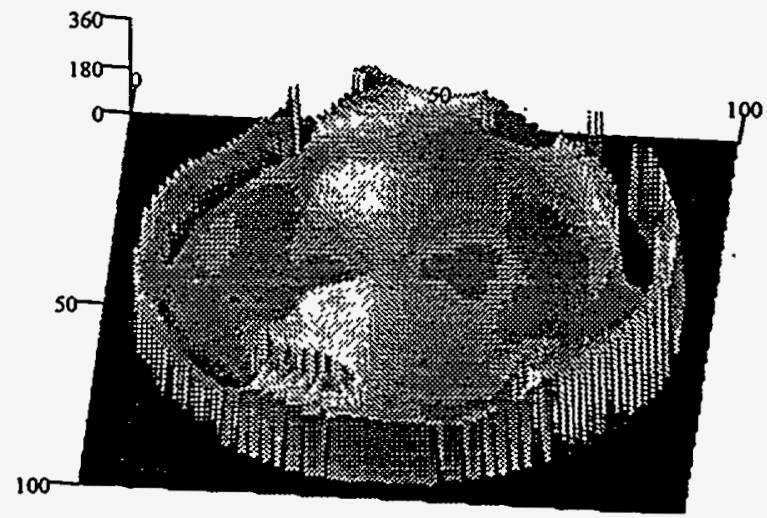

Phase

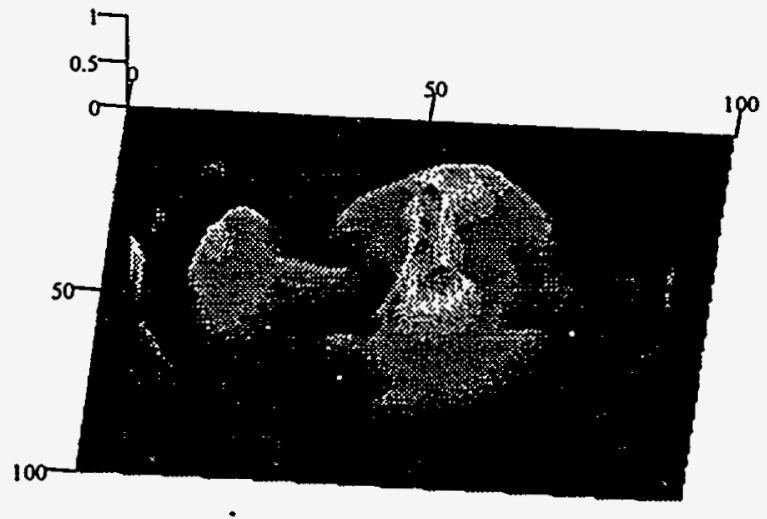

Intensity

Figure 11. 1/2 Dielectric Block. A) Theoretically predicted $(\mathrm{T}=90 \%, \Delta \theta=0.9 \pi)$ and $\mathrm{B})$ experimentally measured amplitude, phase, and intensity. 
A) Gaussian Model

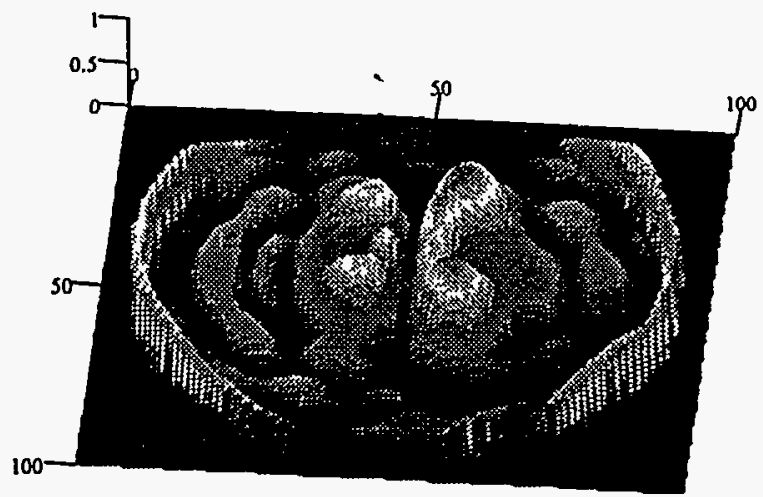

Amplitude

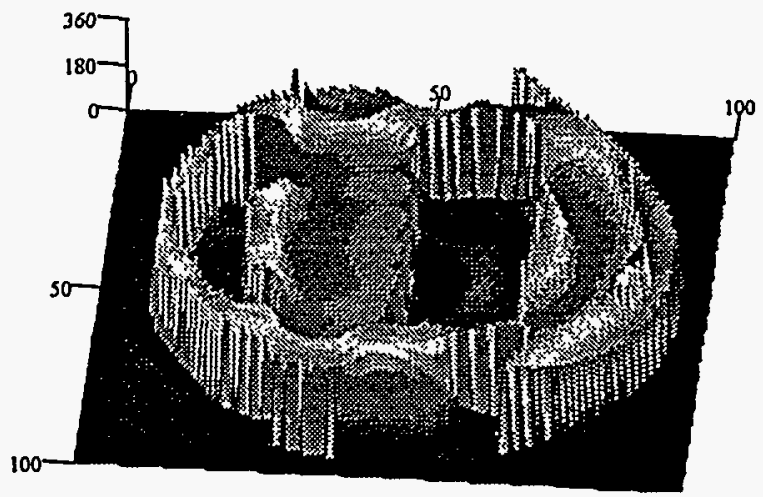

Phase

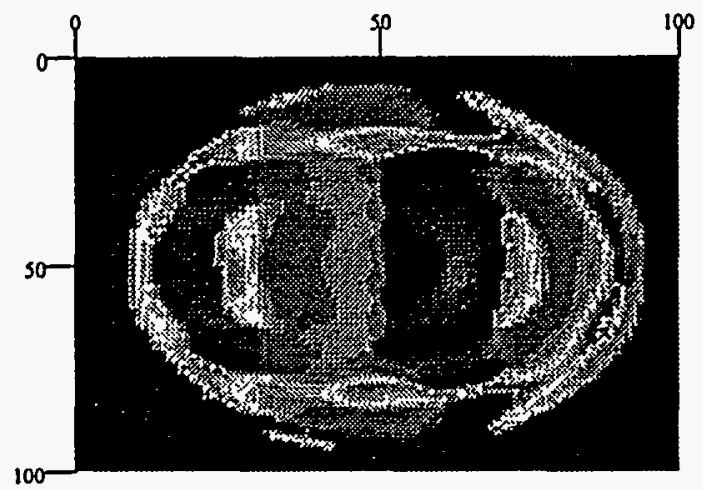

Phase

(Top View)
B) Measured

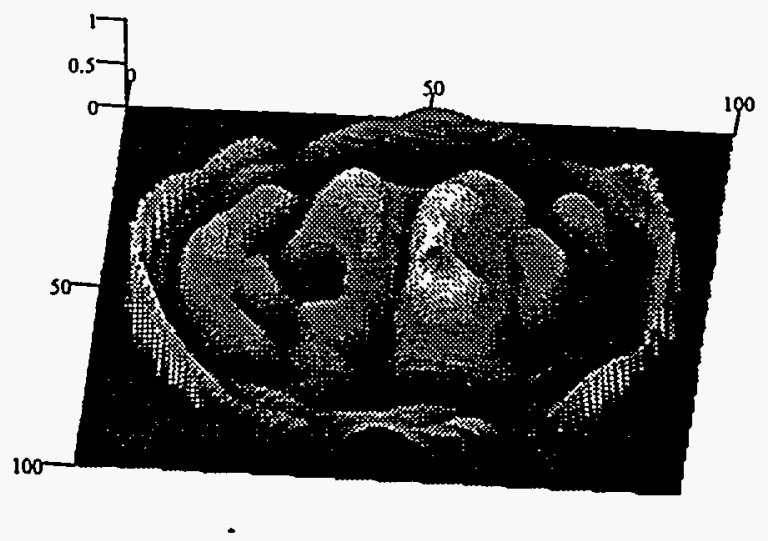

Amplitude

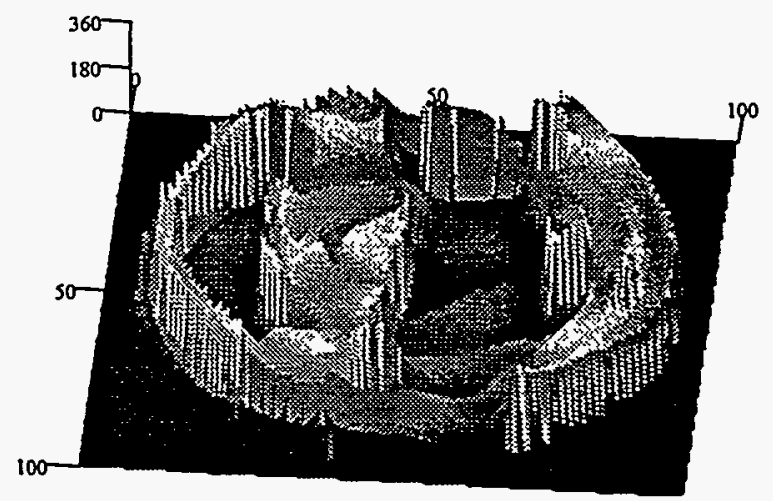

Phase

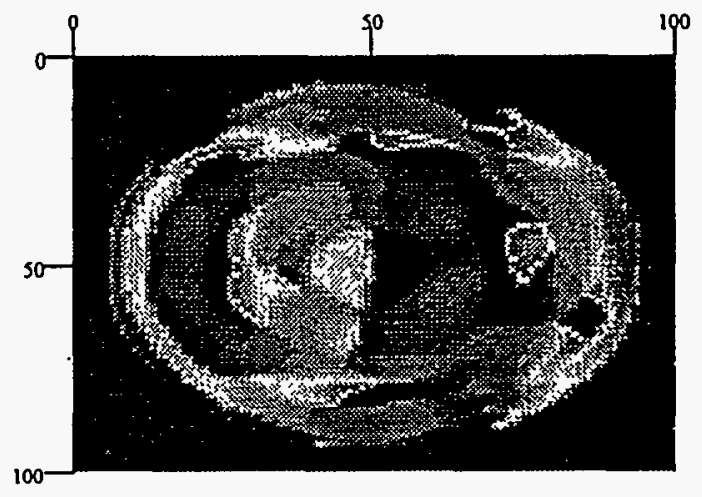

Phase

(Top View)

Figure 12. I/2 Dielectric Block - Diagonalized. A) Theoretically predicted $(T=90 \%, \Delta \theta=0.9 \pi)$ and B) experimentally measured amplitude and phase. Within the limitations imposed by the 36 tern Zernike expansion, the formation of the phase shift discontinuity caused by the microscope slide edge is visible down the center of the plase image. 


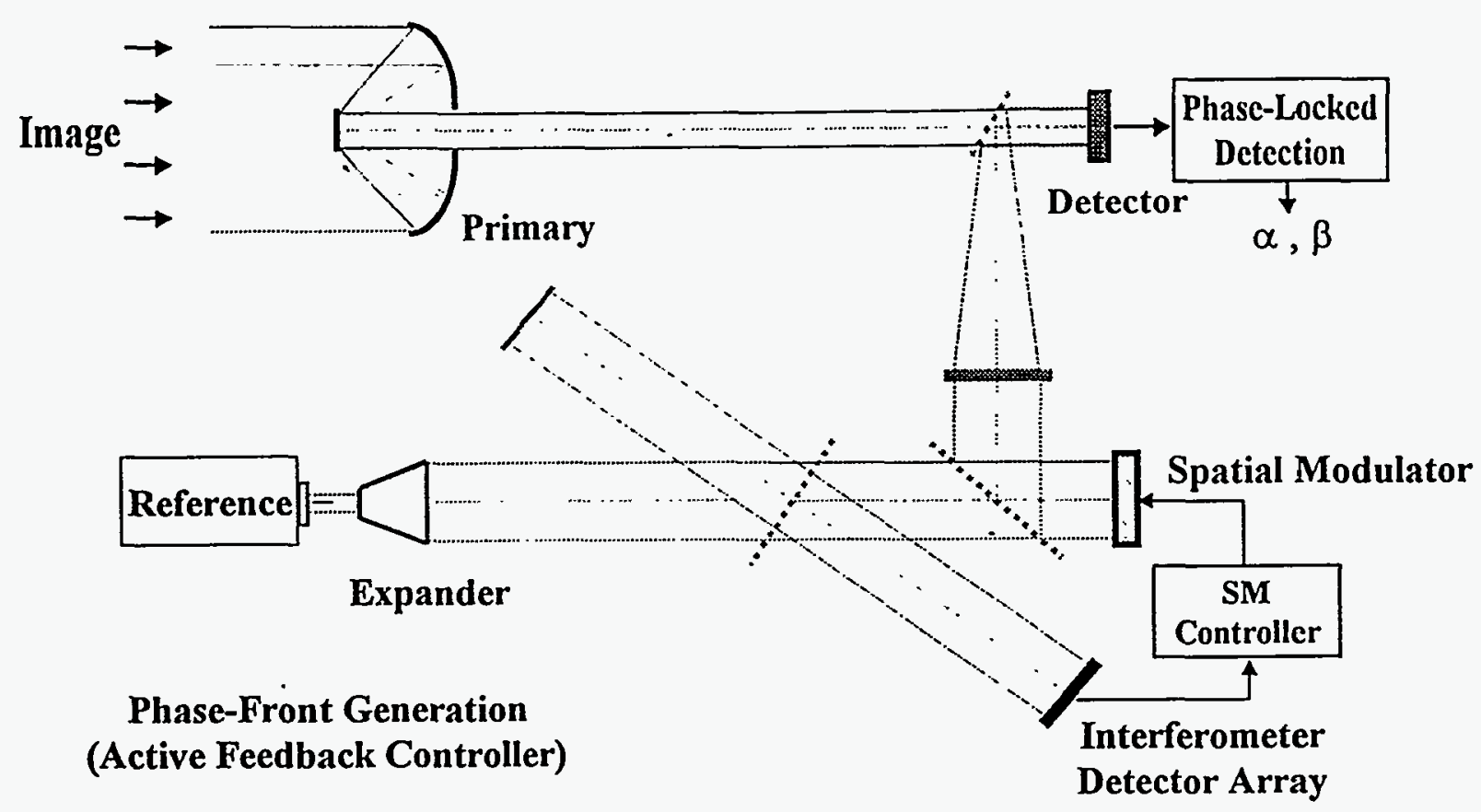

Figure 13. Improved FTH receiver implementation based on knowledge gained from the experimental apparatus. Note that a reflective spatial modulator is used in this example.

\section{Practical Considerations}

An improved FTH receiver implementation, based on knowledge gained from the simple experimental apparatus of Figure 4 is diagramed in Figure 13. Practical consideration is given to active phase-front correction, phase locked detection, pulsed mode correlated double sample, and pure phase basis functions.

\subsection{Active Phase-Front Correction}

The subsystem generating the spatial phase-fronts will require systematic monitoring and calibration for the removal of phase distortion introduced, for example, by alignment error or modulator drift. Inserting an interferometer into the phase-front beam path is one approach to direct wavefront monitoring. Referring to the phase-front generation subsystem of Figure 13, as the spatial modulator (SM) scans through pre-programmed phase functions, the interferometer array monitors the phase-front interference distribution and provides corrective feedback through the SM controller. Note that each SM element must be programmable within a spatial phase-shift of at least $\pm \pi$ (for the wavelength of interest) or larger depending on the feedback algorithm implemented.

\subsection{Phase Locked Detection}

Practical FTH systems must acquire Fourier projected coefficients at a sufficient rate of speed. Detection of coefficients requires the precision measurement of the I.F. phase and magnitude. 


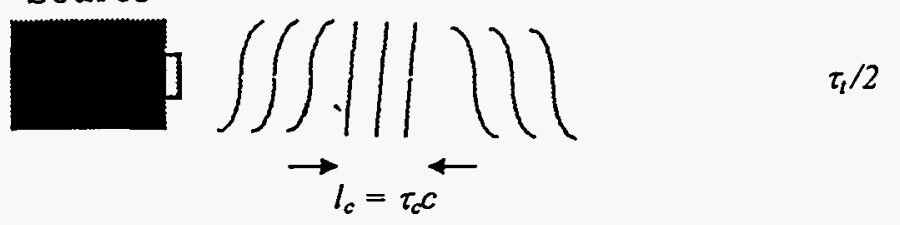

Figure 14. FTH imaging resolution can be limited by the fundamental colherence properties governing laser and RF sources. Correlated sampling algorithms can aid in mitigating the limitations imposed by a source's finite colerence time, $\tau_{c}$.

Precision high-speed acquisition can be accomplished through techniques employing I.F. phaselocking ${ }^{10}$ for measurement of magnitude and phase ( $\alpha$ and $\beta$ ). Phase lock detection will dramatically improve acquisition speed and accuracy over the current experimental configuration (Figure 5).

\subsection{Pulsed Mode Correlated Double Sample}

FTH imaging resolution can be limited by the fundamental coherence properties governing laser and RF sources. The degradation is a manifestation of transverse amplitude and phase fluctuations on the order of the coherence time ${ }^{11}, \tau_{c}$, which is intrinsic to a given coherent source. In essence, source variations cannot be distinguished from image induced modulations, thereby limiting resolution.

What follows is a description of a correlated sampling algorithm that can aid in mitigating the limitations imposed by a source's finite coherence. Correlated double sampling (CDS), as applied to FTH, is the process of removing source fluctuations from the target by correlating the outgoing transmitted pulse to the return, target-modulated pulse, through a common reference. For example, a pulsed transmitter-receiver system acquiring one coefficient $C_{m, n}$ per pulse would implement the following algorithm.

- Measure the Fourier projection coefficient, $C_{X m, n}$, from the basis function $\Phi_{m, n}$ of the outgoing transmitted pulse. The measurement must be performed within the sources coherence time $\tau_{c}$.

- Measure the Fourier projection coefficient, $C_{R m, n}$, from the basis function $\Phi_{m, n}$ of the target-modulated return pulse (after a transit time $\tau_{t}$ ).

- Subtract the common transverse amplitude and phase error:

$$
C_{m, n} \Phi_{m, n}=\left(C_{R m, n}-C_{X m, n}\right) \Phi_{m, n} \rightarrow C_{m, n}=C_{R m, n}-C_{X m, n} .
$$

As both the outgoing and return pulses are compared to a common reference, it is essential that the reference field is properly conditioned (for example, spatially filtered) and stationary over the transit time $\tau_{t}$. Note that as an additional benefit to CDS, any stationary spatial phase-or amplitude structure in the local oscillator is correlated out. 


\subsection{Basis Functions}

FTH receivers, in general, may implement basis functions in which both amplitude and phase are modulated. Complex modulators are practical only if they can achieve sufficiently high modulation rates and are readily calibrated. More efficient are systems in which only the phase is modulated through a refractive or reflective spatial modulator. From Equation 12, pure phase modulation requires the phase function argument

$$
\theta(x, y)=i \ln \left(\frac{1}{\rho^{\prime}(x, y)} \Phi_{R e f}(x, y)\right)
$$

remain real for synthesis with a refractive or reflective spatial modulator. For example, the familiar two-dimensional Cartesian exponential Fourier series ( $x, y$ normalized to a unit square)

$$
\Phi_{m, n}(x, y)=e^{-i 2 \pi(m x+m y)},
$$

possesses the real phase function

$$
\theta(x, y)=2 \pi(m x+n y)
$$

Implementation of Equation 25 suggests a spatial phase modulator generating an $x, y$ phase pattern across the modulator with slope proportional to $m, n$ respectively. Furthermore, as coherent fields are longitudinally periodic, the spatial phase "wraps around" every $2 \pi$, limiting modulation range to $\pm \pi$.

\section{Conclusion}

We have introduced a detection process capable of directly imaging the transverse amplitude and phase of coherent electromagnetic fields. The detection process, Fourier Transform Heterodyne (FTH), incorporates transverse spatial encoding of the local oscillator along with Fourier projection principles for field imaging. Appropriate selection of spatial encoding functions allows image retrieval by way of classic Fourier manipulations. The underlying principles governing FTH imaging were demonstrated via a simple experimental setup based on a HeNe laser and a 69 element spatial phase modulator. The experimental data, when compared to theoretical predictions, do indeed indicate that the basic FTH mechanisms expected are present, validating the concept. 


\section{Appendix A: Heterodyne Phase Projection}

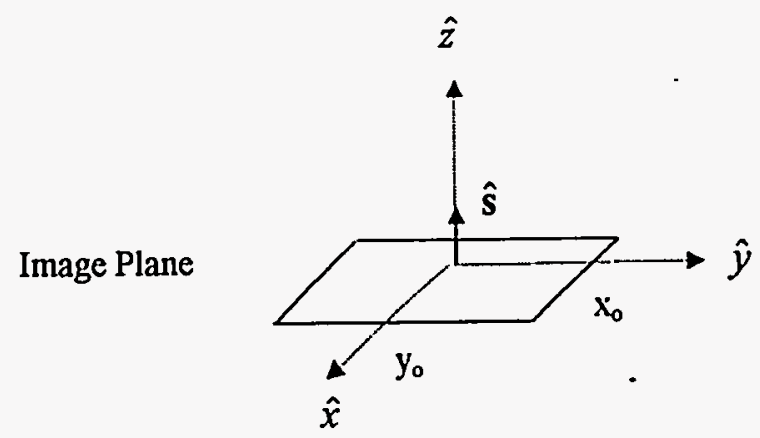

From Section 2, the dependence of the induced detector current $I$, the detector quantum efficiency $\eta$, and signal and local oscillator (1.o.) electromagnetic fields over the detector surface $s$ are represented by

$$
I=k \iint_{s} \eta(\mathbf{E} \times \mathbf{H}) \cdot d \mathbf{s}
$$

where $\mathrm{k}=$ constant, $\mathbf{E}=\mathbf{E}_{\mathbf{s}}+\mathbf{E}_{10}$, and $\mathbf{H}=\mathbf{H}_{\mathbf{s}}+\mathbf{H}_{10}$ (signal + local oscillator). Expanding and projecting the field components through the surface of the detector

$$
\left|\begin{array}{ccc}
\hat{x} & \hat{y} & \hat{z} \\
E_{x} & E_{y} & E_{z} \\
H_{x} & H_{y} & H_{z}
\end{array}\right|=\left(E_{y} H_{z}-E_{z} H_{y}\right) \hat{x}-\left(E_{x} H_{z}-E_{z} H_{x}\right) \hat{y}+\left(E_{x} H_{y}-H_{x} E_{y}\right) \hat{z}
$$

results in

$$
I=k \int_{x_{0}} \int_{y_{0}} \eta(x, y)\left(E_{x} H_{y}-H_{x} E_{y}\right) d x d y
$$

Assuming the fields are transverse electromagnetic (TEM) and carrying only the $x$ axis of polarization (along $E_{x}$ )

$$
\begin{gathered}
I=k \iint_{x_{0} y_{0}} \eta(x, y)\left(E_{s_{x}}+E_{l o_{x}}\right)\left(H_{s_{y}}+H_{b_{y}}\right) d x d y \\
=k \iint_{x_{0} y_{o}} \eta(x, y)\left(E_{s_{x}} H_{s_{y}}+E_{l b_{x}} H_{l o_{y}}+E_{s_{x}} H_{l b_{y}}+E_{l o_{x}} H_{s_{y}}\right) d x d y .
\end{gathered}
$$


If the signal is now bandpassed at the heterodyne intermediate frequency (I.F.), the DC terms are filtered out and the resulting I.F. signal becomes

$$
I_{I F}=k \iint_{x_{0} y_{0}} \eta(x, y)\left(E_{s_{x}} H_{l o_{y}}+E_{l o_{x}} H_{s_{y}}\right) d x d y
$$

Furthermore, since the fields are TEM then $H_{y}=\sqrt{\frac{\varepsilon}{\mu}} E_{x}$ and

$$
I_{I F}=2 k \sqrt{\frac{\varepsilon}{\mu}} \iint_{x_{0} y_{0}} \eta(x, y) E_{s_{x}} E_{l o_{x}} d x d y .
$$

A similar term for the $E_{y}$ polarization can be derived.

For signal and local oscillator fields of the form

$$
\begin{aligned}
& E_{s}(x, y)=\rho(x, y)_{s} e^{-i \omega t-i \phi(x, y)}, \\
& E_{l o}(x, y)=\rho(x, y)_{l o} e^{-i \omega^{\prime} t-i \theta(x, y)},
\end{aligned}
$$

results in

$$
\begin{aligned}
& I_{i f}=2 k \sqrt{\frac{\varepsilon}{\mu}} \operatorname{Re}\left[e^{i \Delta \omega t} \iint_{s} \eta(x, y) \rho(x, y)_{s} \rho(x, y)_{l o} e^{-i \phi(x, y)} e^{i \theta(x, y)} d x d y\right] \\
& =2 k \sqrt{\frac{\varepsilon}{\mu}} \operatorname{Re}\left[e^{i \Delta \omega t} \beta e^{i \alpha}\right] \\
& =2 k \sqrt{\frac{\varepsilon}{\mu}} \beta \cos (\Delta \omega t+\alpha)
\end{aligned}
$$

where $\Delta \omega=\left|\omega-\omega^{\prime}\right|$ is the heterodyne intermediate frequency. 


\section{Appendix B: Zernike Polynomials}

$Z_{0}=1$

$Z_{1}=r \cos (\theta)$

$Z_{2}=r \sin (\theta)$

$Z_{3}=2 r^{2}-1$

$Z_{4}=r^{2} \cos (2 \theta)$

$Z_{\mathrm{s}}=r^{2} \sin (2 \theta)$

$Z_{6}=\left(3 r^{2}-2\right) r \cos (\theta)$

$Z_{7}=\left(3 r^{2}-2\right) r \sin (\theta)$

$Z_{8}=6 r^{4}-6 r^{2}+1$

$Z_{9}=r^{3} \cos (3 \theta)$

$Z_{10}=r^{3} \sin (3 \theta)$

$Z_{11}=\left(4 r^{2}-3\right) r^{2} \cos (2 \theta)$

$Z_{12}=\left(4 r^{2}-3\right) r^{2} \sin (2 \theta)$

$Z_{13}=\left(10 r^{4}-12 r^{2}+3\right) r \cos (\theta)$

$Z_{14}=\left(10 r^{4}-12 r^{2}+3\right) r \sin (\theta)$

$Z_{15}=20 r^{6}-30 r^{4}+12 r^{2}-1$

$Z_{16}=r^{4} \cos (4 \theta)$

$Z_{17}=r^{4} \sin (4 \theta)$
$Z_{18}=\left(5 r^{2}-4\right) r^{3} \cos (3 \theta)$

$Z_{19}=\left(5 r^{2}-4\right) r^{3} \sin (3 \theta)$

$Z_{20}=\left(15 r^{4}-20 r^{2}+6\right) r^{2} \cos (2 \theta)$

$Z_{21}=\left(15 r^{4}-20 r^{2}+6\right) r^{2} \sin (2 \theta)$

$Z_{22}=\left(35 r^{6}-60 r^{4}+30 r^{2}-4\right) r \cos (\theta)$

$Z_{23}=\left(35 r^{6}-60 r^{4}+30 r^{2}-4\right) r \sin (\theta)$

$Z_{24}=\left(70 r^{8}-140 r^{6}+90 r^{4}-20 r^{2}+1\right)$

$Z_{25}=r^{5} \cos (5 \theta)$

$Z_{26}=r^{5} \sin (5 \theta)$.

$Z_{27}=\left(6 r^{2}-5\right) r^{4} \cos (4 \theta)$

$Z_{28}=\left(6 r^{2}-5\right) r^{4} \sin (4 \theta)$

$Z_{29}=\left(21 r^{4}-30 r^{2}+10\right) r^{3} \cos (3 \theta)$

$Z_{30}=\left(21 r^{4}-30 r^{2}+10\right) r^{3} \sin (3 \theta)$

$Z_{31}=\left(56 r^{6}-105 r^{4}+60 r^{2}-10\right) r^{2} \cos (2 \theta)$

$Z_{32}=\left(56 r^{6}-105 r^{4}+60 r^{2}-10\right) r^{2} \sin (2 \theta)$

$Z_{33}=\left(126 r^{8}-280 r^{6}+210 r^{4}-60 r^{2}+5\right) r \cos (\theta)$

$Z_{34}=\left(126 r^{8}-280 r^{6}+210 r^{4}-60 r^{2}+5\right) r \sin (\theta)$

$Z_{35}=\left(252 r^{10}-630 r^{8}+560 r^{6}-210 r^{4}+30 r^{2}-1\right)$ 
Appendix C: Amplitude and phase progression, $\Delta \theta=0$ to $2 \pi$ over 36-term (diagonalized) expansion window, for $1 / 2$ dielectric block $(T=90 \%$, Gaussian model).

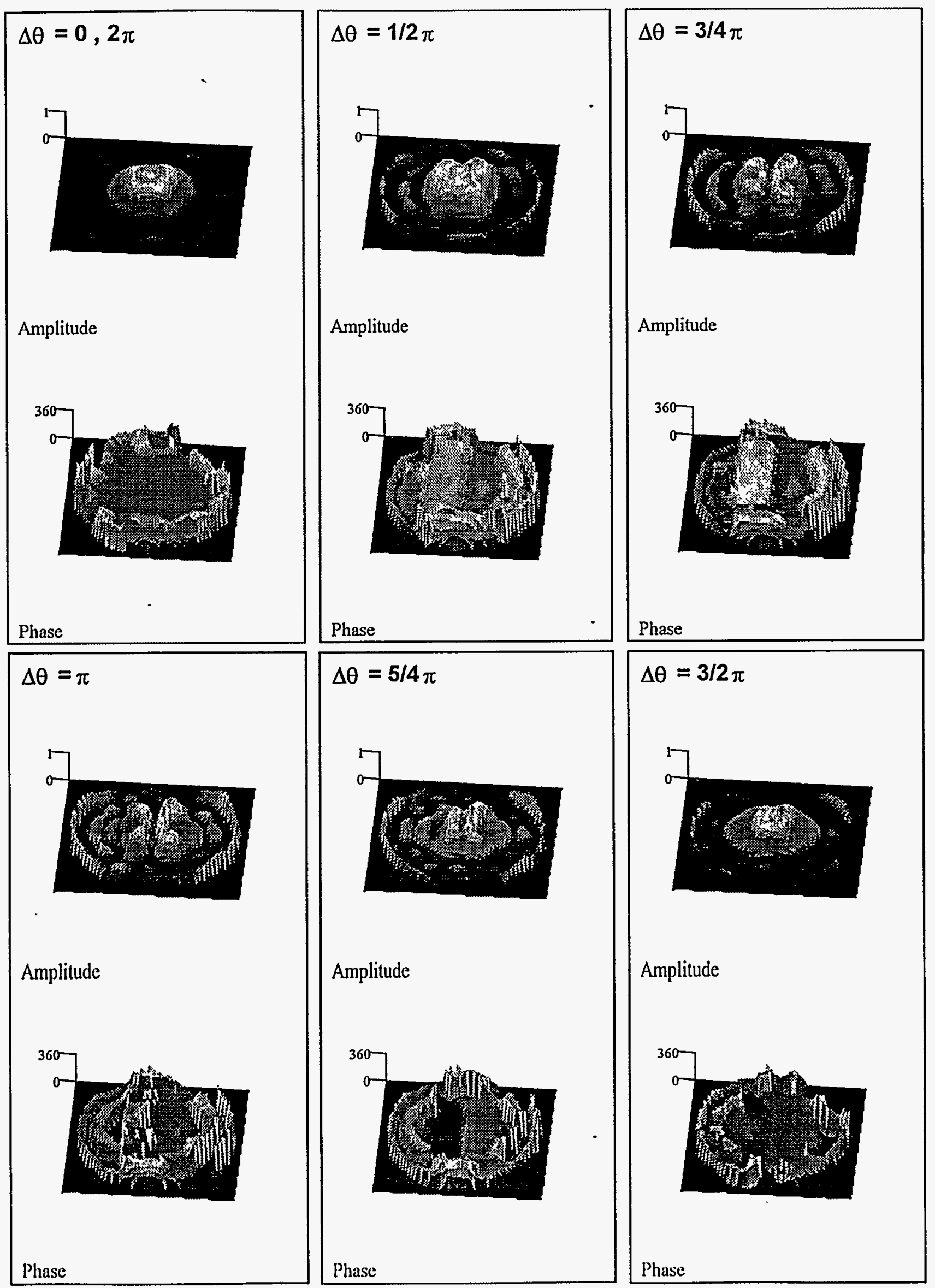




\section{References}

${ }^{1}$ M. C. Roggemann and B. Welsh, Imaging Through Turbulence, CRC Press, 1996.

${ }^{2}$ J. W. Goodman, Introduction to Fourier Optics, McGraw-Hill, 1968.

${ }^{3}$ G. O. Reynolds, J. B. DeViles, G. B. Parrent and Brian J. Thompson, Physical Optics Notebook Tutorials In Fourier Optics, SPIE and American Institute of Physics, pp. 94-96, 1989.

${ }^{4}$ J. D. Jackson, Classical Electrodynamics, John Wiley \& Sons, $2^{\text {nd }}$ edition, 1975.

${ }^{5}$ S. C. Cohen, "Heterodyne Detection: Phase Front Alignment, Beam Spot Size, and Detector Uniformity," Applied Optics, vol. 14, no. 8, pp. 9153-1958, Aug. 1975.

${ }^{6}$ S. Fowler, G. W. Kamerman, and G. Lawson, "Analysis of Heterodyne Efficiency for Coherent Laser Radars," SPIE Applied Laser Radar Technology, vol. 1936, pp. 137-146, 1993.

${ }^{7}$ J. Y. Wang and D. E. Silva, "Wave-Front Interpretation With Zernike Polynomials," Applied Optics, vol. 19, no. 9, pp. 1510-1513, May 1980.

${ }^{8} \mathrm{~K}$. Chang, Handbook of Microwave and Optical Components Vol. 3, pp. 28-32, John Wiley \& Sons, 1990.

${ }^{9}$ O. K. Ersoy, Fourier-Related Transforms, Fast Algorithms and Applications, Prentice Hall, 1997.

${ }^{10}$ F. M. Gardner, Phaselock Techniques, John Wiley \& Sons, 1979.

${ }^{11}$ J. W. Goodman, Statistical Optics, John Wiley \& Sons, 1985. 